\title{
The Circumstellar Disk HD 169142: Gas, Dust, and Planets Acting in Concert?*
}

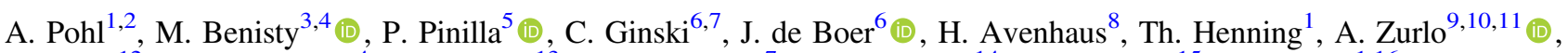 \\ A. Boccaletti ${ }^{12}$, J.-C. Augereau ${ }^{4}$, T. Birnstiel ${ }^{13}$ (D), C. Dominik ${ }^{7}$ (D), S. Facchini ${ }^{14}$ (D), D. Fedele ${ }^{15}$, M. Janson ${ }^{1,16}$ (D), M. Keppler ${ }^{1}$, \\ Q. Kral $^{12,17}$, M. Langlois ${ }^{9,18}$, R. Ligi ${ }^{9}$, A.-L. Maire ${ }^{1}$ (D) F. Ménard ${ }^{4}$ (D) M. Meyer ${ }^{8}$ (D) C. Pinte ${ }^{4}$ (D) S. P. Quanz ${ }^{8}$ (D) J.-F. Sauvage ${ }^{19}$, \\ É. Sezestre $^{4}$, T. Stolker ${ }^{7}$ (D) J. Szulágyi ${ }^{8}$, R. van Boekel ${ }^{1}$, G. van der Plas ${ }^{4}$ (D), M. Villenave ${ }^{4}$, A. Baruffolo ${ }^{20}$ (D), P. Baudoz ${ }^{12}$, \\ D. Le Mignant ${ }^{9}$, D. Maurel ${ }^{4}$, J. Ramos ${ }^{1}$, and L. Weber ${ }^{21}$ \\ ${ }^{1}$ Max-Planck-Institute for Astronomy, Königstuhl 17, D-69117 Heidelberg, Germany; pohl@mpia.de \\ ${ }^{2}$ Heidelberg University, Institute of Theoretical Astrophysics, Albert-Ueberle-Str. 2, D-69120 Heidelberg, Germany \\ ${ }^{3}$ Unidad Mixta Internacional Franco-Chilena de Astronomía, CNRS/INSU UUMI 3386 and Departamento de Astronomía, \\ Universidad de Chile, Casilla 36-D, Santiago, Chile \\ ${ }^{4}$ Univ. Grenoble Alpes, CNRS, IPAG, F-38000 Grenoble, France \\ ${ }^{5}$ Department of Astronomy/Steward Observatory, University of Arizona, 933 North Cherry Avenue, Tucson, AZ 85721, USA \\ ${ }^{6}$ Leiden Observatory, Leiden University, PO Box 9513, 2300 RA, Leiden, The Netherlands \\ ${ }^{7}$ Anton Pannekoek Institute for Astronomy, University of Amsterdam, Science Park 904, 1098 XH Amsterdam, The Netherlands \\ ${ }^{8}$ Institute for Astronomy, ETH Zurich, Wolfgang-Pauli-Strasse 27, 8093 Zurich, Switzerland \\ ${ }^{9}$ Aix Marseille Université, CNRS, LAM (Laboratoire d'Astrophysique de Marseille) UMR 7326, F-13388, Marseille, France \\ ${ }^{10}$ Núcleo de Astronomía, Facultad de Ingeniería, Universidad Diego Portales, Av. Ejercito 441, Santiago, Chile \\ ${ }^{11}$ Departamento de Astronomía, Universidad de Chile, Casilla 36-D, Santiago, Chile \\ ${ }^{12}$ LESIA, Observatoire de Paris, PSL Research University, CNRS, Sorbonne Universités, UPMC Univ. Paris 06, Univ. Paris Diderot, Sorbonne Paris Cité, 5 place \\ Jules Janssen, F-92195 Meudon, France \\ ${ }^{13}$ University Observatory, Faculty of Physics, Ludwig-Maximilians-Universität München, Scheinerstr. 1, D-81679 München, Germany \\ ${ }_{14}$ Max-Planck-Institut für Extraterrestrische Physik, Giessenbachstrasse 1, D-85748 Garching, Germany \\ ${ }^{15}$ INAF-Osservatorio Astrofisico di Arcetri, L.go E. Fermi 5, I-50125 Firenze, Italy \\ ${ }^{16}$ Department of Astronomy, Stockholm University, AlbaNova University Center, SE-10691 Stockholm, Sweden \\ ${ }^{17}$ Institute of Astronomy, University of Cambridge, Madingley Road, Cambridge CB3 0HA, UK \\ ${ }^{18}$ CRAL, UMR 5574, CNRS, Université Lyon 1, 9 avenue Charles André, F-69561 Saint Genis Laval Cedex, France
Institute of Astronomy, University of Cambridge, Madingley Road, Cambridge CB3 OHA, UK \\ ${ }^{19}$ ONERA, Optics Department, BP 72, F-92322 Chatillon, France \\ ${ }^{20}$ INAF-Osservatorio Astronomico di Padova, Vicolo dell'Osservatorio 5, I-35122 Padova, Italy \\ ${ }^{21}$ Geneva Observatory, University of Geneva, Chemin des Maillettes 51, 1290 Versoix, Switzerland \\ Received 2017 July 27; revised 2017 October 10; accepted 2017 October 17; published 2017 November 16
}

\begin{abstract}
HD 169142 is an excellent target for investigating signs of planet-disk interaction due to previous evidence of gap structures. We perform $J$-band $(\sim 1.2 \mu \mathrm{m})$ polarized intensity imaging of HD 169142 with VLT/SPHERE. We observe polarized scattered light down to 0 !"16 $(\sim 19 \mathrm{au})$ and find an inner gap with a significantly reduced scattered-light flux. We confirm the previously detected double-ring structure peaking at 0 ". 18 ( $\sim 21$ au) and 0 ". 56 ( $\sim 66 \mathrm{au})$ and marginally detect a faint third gap at 0!"70-0! 73 ( $\sim 82-85 \mathrm{au})$. We explore dust evolution models in a disk perturbed by two giant planets, as well as models with a parameterized dust size distribution. The dust evolution model is able to reproduce the ring locations and gap widths in polarized intensity but fails to reproduce their depths. However, it gives a good match with the ALMA dust continuum image at 1.3 mm. Models with a parameterized dust size distribution better reproduce the gap depth in scattered light, suggesting that dust filtration at the outer edges of the gaps is less effective. The pileup of millimeter grains in a dust trap and the continuous distribution of small grains throughout the gap likely require more efficient dust fragmentation and dust diffusion in the dust trap. Alternatively, turbulence or charging effects might lead to a reservoir of small grains at the surface layer that is not affected by the dust growth and fragmentation cycle dominating the dense disk midplane. The exploration of models shows that extracting planet properties such as mass from observed gap profiles is highly degenerate.
\end{abstract}

Key words: planet-disk interactions - protoplanetary disks - radiative transfer - scattering - techniques: polarimetric

\section{Introduction}

About two decades ago, our own solar system was the only available laboratory to test models of planet formation. Today, we know that planetary systems are common around other stars and that their architectures are very diverse. The initial conditions and evolution of protoplanetary disks, where planets form, must have a direct influence on most

\footnotetext{
* Based on observations collected at the European Organisation for Astronomical Research in the Southern Hemisphere under ESO program 095.C-0273.
}

fundamental properties of their planetary systems (Mordasini et al. 2012, 2016). It is therefore essential to improve our knowledge of the structure of protoplanetary disks by observing and studying them at high spatial scales and with various tracers that enable us to characterize different disk regions. This, indirectly, can constrain the physical processes that influence the disk evolution (e.g., gap opening by a planet, dust growth and settling, photo-evaporation). Even with the advent of a new generation of extreme adaptive optics instruments, the detection of forming planets within their host disks is still challenging, but one can look for indirect 
signatures of planet formation, such as the imprints that it leaves on the disk.

In recent years, high-resolution images of protoplanetary disks have been published, both in scattered light that traces the (sub-)micron-sized dust particles in the upper disk layers and in the (sub)millimeter ((sub-)mm) regime that traces larger dust grains ( $\mathrm{mm}$ and centimeter-sized $(\mathrm{cm})$ grains), while the bulk mass is not directly observable. While for a long time protoplanetary disks were thought to be smooth and continuous, a variety of small-scale features are now frequently detected in these images and seem rather common, if not ubiquitous. Large cavities ( $\sim$ few tens of au) are detected in a number of objects (e.g., Williams \& Cieza 2011), the transition disks, that often have spectral energy distributions (SEDs) with a clear dip in the mid-infrared (MIR), indicating a lack of dust in the inner regions (Strom et al. 1989). Smaller cavities and gaps in the inner au are also present (Menu et al. 2015) but cannot be easily directly imaged, nor do they leave a clear imprint on the SED. Multiple-arm spiral features are observed, mostly in scattered light (Muto et al. 2012; Garufi et al. 2013; Grady et al. 2013; Avenhaus et al. 2014; Benisty et al. 2015; Stolker et al. 2016; Benisty et al. 2017), but also more recently in the sub-mm wavelength range (CO gas lines: Christiaens et al. 2014; Tang et al. 2017; continuum emission: Pérez et al. 2016). These spiral arms have large opening angles, and their origin is still not fully understood. The presence of one or more rings and gaps in disks seems to be quite a common feature; they are found in both young (e.g., HL Tau; ALMA Partnership et al. 2015; Carrasco-González et al. 2016) and rather old systems (e.g., TW Hya; Rapson et al. 2015; Andrews et al. 2016; Tsukagoshi et al. 2016; van Boekel et al. 2017) and around stars of very different spectral types (e.g., de Boer et al. 2016; Ginski et al. 2016; van der Plas et al. 2017). Various mechanisms have been proposed in the literature that can be assigned to three main categories: structures caused by fluid dynamics, dust evolution effects, and planet-disk perturbations. More precisely, these possibilities include zonal flows from magneto-rotational instability (e.g., Simon \& Armitage 2014; Béthune et al. 2016), gap/bump structures in the surface density close to the dead-zone outer edge (e.g., Flock et al. 2015; Pinilla et al. 2016; Ruge et al. 2016), efficient particle growth at condensation fronts near ice lines or a depletion of solid material between ice lines (Zhang et al. 2015; Pinilla et al. 2017; Stammler et al. 2017), aggregate sintering zones (Okuzumi et al. 2016), secular gravitational instabilities (Youdin 2011; Takahashi \& Inutsuka 2014), or planet-disk interactions (e.g., Zhu et al. 2011, 2012; Dong et al. 2015, 2016; Rosotti et al. 2016). Finally, dips or dark regions can be interpreted as shadows by inner disk material (e.g., Marino et al. 2015; Pinilla et al. 2015b; Stolker et al. 2016; Canovas et al. 2017).

We focus on a multiple-ring system in this study: specifically, the $\sim 6_{-3}^{+6}$ Myr old Herbig A5/A8 star HD 169142 (Dunkin et al. 1997; Grady et al. 2007), located at a distance ${ }^{22}$ of $d=117 \pm 4$ pc (Gaia Collaboration et al. 2016). With this new distance of $117 \mathrm{pc}$, the star is intrinsically less luminous by a factor of $\sim 0.65$. The age estimate by Grady et al. (2007) is based on Hertzsprung-Russell (HR) placement of the companion 2MASS 18242929-2946559. Moving this star down in the HR diagram (Figure 9 in Grady et al. 2007) leads to a revised age

\footnotetext{
22 Note that we are using the revised value by Gaia, while most of the papers in the literature use $d=145 \mathrm{pc}$.
}

estimate of $\sim 10$ Myr. Its SED shows a strong infrared excess indicating a young gas-rich disk with many emission-line features (Kama et al. 2016; Riviere-Marichalar et al. 2016; Seok \& $\mathrm{Li}$ 2017) and a clear dip of emission in the infrared regime (Grady et al. 2007; Meeus et al. 2010), qualifying it as a transition disk. The near-infrared (NIR) flux indicates the presence of hot dust close to the sublimation radius, resolved by NIR interferometric observations (Lazareff et al. 2017). HD 169142 still experiences gas accretion onto the star, with estimates of the mass accretion rate varying between 0.7 and $2.7 \times 10^{-9} M_{\odot} \mathrm{yr}^{-1}$ (Grady et al. 2007; Wagner et al. 2015). Garufi et al. (2017) noted that HD 169142 has a reduced NIR excess compared to continuous Herbig disks or those hosting spirals. The NIR and MIR fluxes were also found to vary by up to $\sim 45 \%$ over a temporal baseline of $10 \mathrm{yr}$, indicating strong variability in the innermost regions (Wagner et al. 2015).

The outer disk has a low inclination $\left(i=13^{\circ}, \mathrm{PA}=5^{\circ}\right)$, as derived by $\mathrm{CO} \mathrm{mm}$ observations (Raman et al. 2006; Panić et al. 2008) and confirmed with high-contrast imaging in the NIR (Quanz et al. 2013; Momose et al. 2015; Monnier et al. 2017). These images show, from small to larger separations from the star, a wide inner cavity, a bright (unresolved) ring, a second wide gap, and an outer disk that extends up to 1 ".7. The inner cavity appears devoid of small dust grains, while the second gap is not. Observations with the Atacama Large Millimeter Array (ALMA) at $1.3 \mathrm{~mm}$, obtained with a resolution of 0 ". $28 \times 0$ " 18 , also show two rings $(0$ !" $17-0$ ". 28 and 0!" 48-0!" 64) and a gap between them (Fedele et al. 2017). The mm continuum extends up to $0 . " 64$, while the gas extends up to twice as far. The channel maps of the 2-1 line transition of the three $\mathrm{CO}$ isotopologues reveal the presence of gas inside the dust gaps. Model fitting provides a drop in the gas surface density by a factor of 30-40. The two rings are also detected in Very Large Array (VLA) observations at longer wavelengths (7 mm; Osorio et al. 2014; Macías et al. 2017), and the azimuthally averaged radial intensity profiles indicate the marginal detection of a new gap at $\sim 0$. 7 , very close to the CO ice line (Macías et al. 2017). In addition to the disk features, a candidate massive companion was proposed, slightly inside the inner ring, at a separation of $\sim 0$ ". 11 and $\sim 0$ ". 16, respectively (Biller et al. 2014; Reggiani et al. 2014). Osorio et al. (2014) reported the detection of a compact $7 \mathrm{~mm}$ emission source with VLA external to the inner ring. The detection of pointlike structures in the context of potential planetary companions is discussed further in Ligi et al. (2017).

In this paper, we report new polarized differential images of HD 169142 obtained in the J-band with the Spectro-Polarimeter High-contrast Exoplanet REsearch instrument (SPHERE; Beuzit et al. 2008) at the Very Large Telescope (VLT), complemented with ALMA continuum data from Fedele et al. (2017). We investigate whether the observed rings can be explained by the trapping of dust particles as a consequence of the presence of two planets. This study on HD 169142 serves as a prototype in which it is demonstrated that multiwavelength observations are needed to constrain the dust size distribution and physical mechanisms at work in the disk. The paper is organized as follows. Section 2 describes the observations and data processing. Section 3 reports on the detected disk features, Section 4 provides a physical disk model, and, in Section 5, we discuss our findings. 

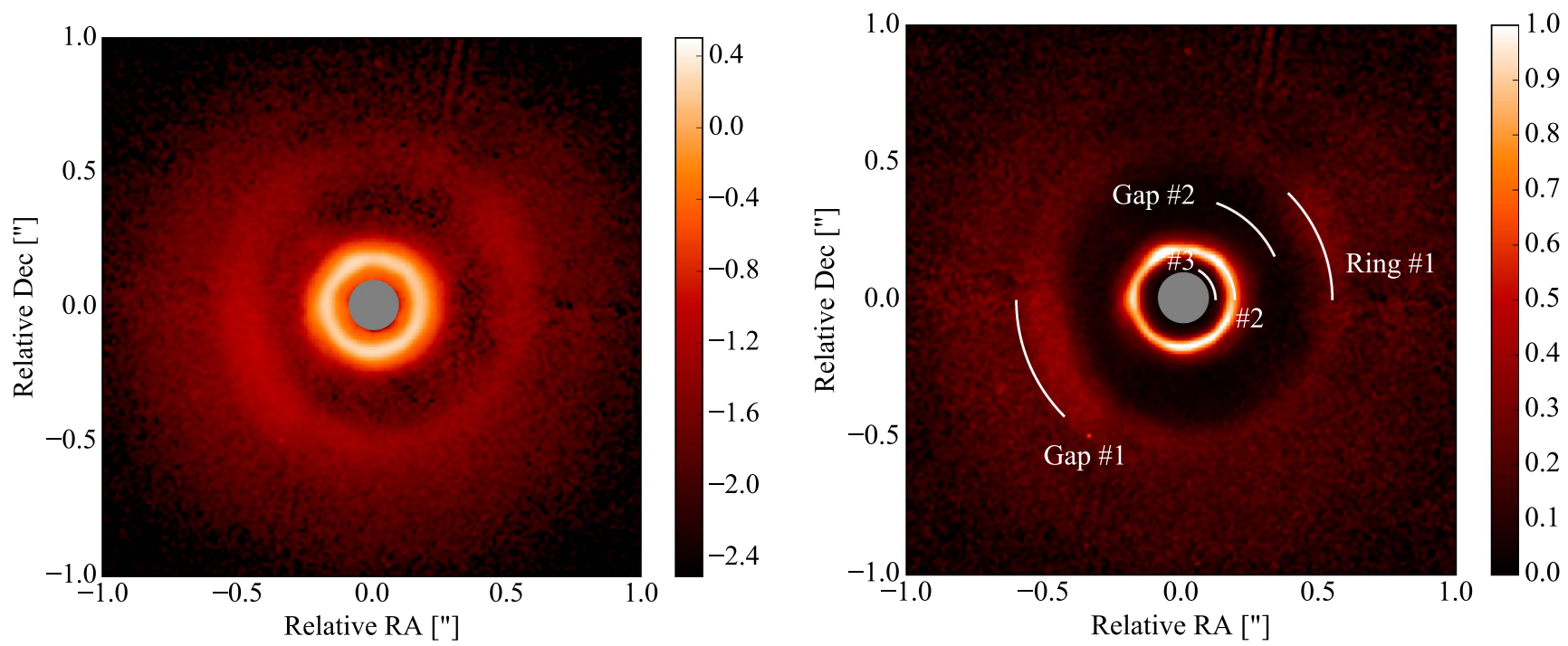

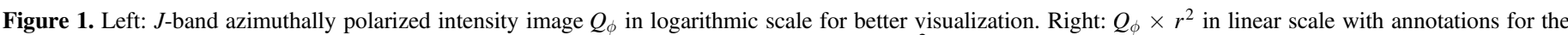

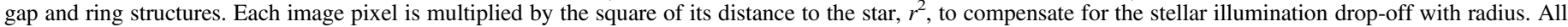

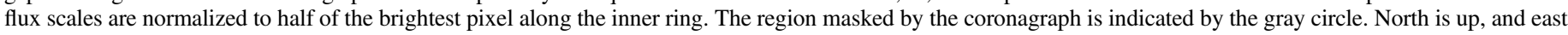
is toward the left.

\section{Observations and Data Reduction}

The observations were obtained at the VLT at Cerro Paranal, Chile, on 2015 May 2 with the SPHERE instrument. SPHERE is equipped with an extreme adaptive optics (AO) system (Fusco et al. 2006; Petit et al. 2014; Fusco et al. 2014) that feeds three science channels allowing for high-angularresolution and high-contrast imaging, spectroimaging, and/or polarimetry at visible and NIR wavelengths. The observations were obtained through the Guaranteed Time program. HD 169142 was observed in the $J$-band filter $\left(\lambda_{0}=1.258\right.$, $\Delta \lambda=0.197 \mu \mathrm{m})$ using the polarimetric imaging mode of the infrared dual-band imager and spectrograph (IRDIS; Dohlen et al. 2008; Langlois et al. 2014), with a plate scale of 12.25 mas pixel $^{-1}$ (Maire et al. 2016) and a 145 mas diameter coronagraphic focal mask (N_ALC_YJ_S, inner working angle (IWA) of 0."08; Boccaletti et al. 2008). HD 169142 was observed for $\sim 53$ minutes on-source under moderate AO conditions (seeing of 0. .9). The analysis of the reference pointspread function (PSF) that is estimated from a noncoronagraphic total intensity measurement shows that the observations reach a 33.8 mas $\times 40.8$ mas resolution $(\mathrm{FWHM}$ along the $x$ and $y$ directions) and a Strehl ratio of $56 \%$.

We observed HD 169142 using the polarimetric differential imaging technique (PDI; e.g., Kuhn et al. 2001; Apai et al. 2004) that measures the linear polarization of the light scattered by dust grains in the disk and enables one to efficiently remove the unpolarized contribution, including that from the star. This allows us to image, with high contrast, the polarized signal from the disk. In this mode, the instrument splits the beam into two orthogonal polarization states. The control of the polarization orientation was performed with a half-wave plate that was set to four positions shifted by 22.5 in order to construct a set of linear Stokes images. We reduce the data according to the double-difference method (Kuhn et al. 2001) and obtain the Stokes parameters $Q$ and $U$. If we assume that there is only one scattering event for each photon, the scattered light from a circumstellar disk seen at a low inclination angle is expected to be linearly polarized in the azimuthal direction. It is therefore convenient to describe the polarization vector field in polar coordinates (Schmid et al. 2006; Avenhaus et al. 2014). We therefore define the polarcoordinate Stokes parameters $Q_{\phi}, U_{\phi}$ as

$$
\begin{gathered}
Q_{\phi}=+Q \cos (2 \phi)+U \sin (2 \phi,), \\
U_{\phi}=-Q \sin (2 \phi)+U \cos (2 \phi),
\end{gathered}
$$

where $\phi$ is the position angle of the location of interest $(x, y)$ with respect to the star location. In this coordinate system, the azimuthally polarized flux appears as a positive signal in the $Q_{\phi}$ image, whereas the $U_{\phi}$ image remains free of disk signal and can be used as an estimate of the residual noise in the $Q_{\phi}$ image (Schmid et al. 2006). This is only valid for disks with face-on geometry, since multiple scattering effects in inclined disks can cause a considerable physical signal in $U_{\phi}$ (e.g., T Cha; Pohl et al. 2017). The correction for instrumental polarization is done using a $U_{\phi}$ minimization by subtracting scaled versions of the total intensity frame from the Stokes $Q$ and $U$ frames. The final data images are corrected for the true north (by rotating them by 1.775 in the counterclockwise direction; Maire et al. 2016). We do not attempt to perform an absolute flux calibration of our images due to the inherent problems with measuring flux in PDI images.

\section{Polarized Intensity Images}

Figures 1 and 10 (Appendix A) show the polarized scatteredlight images $Q_{\phi}$ and $U_{\phi}$, respectively, obtained in the $J$ band. The $U_{\phi}$ image contains very low signal, suggesting that the assumption of single scattering is valid (cf. Canovas et al. 2015). Figure 1 is similar to previously published scatteredlight images of HD 169142, in particular those of Momose et al. (2015) and Monnier et al. (2017), but it brings the highest signal-to-noise ratio view of the inner ring. It shows a number of features. We detect, from outside in:

(a) A faint gap (Gap \#1) at $\sim 0$ !"70-0."73 (81-85 au). Beyond this radius, the image shows diffuse scattered light up to $\sim 1$ ". $5(\sim 176 \mathrm{au})$. The marginal detection of this gap can be seen in the normalized, azimuthally averaged radial profile 

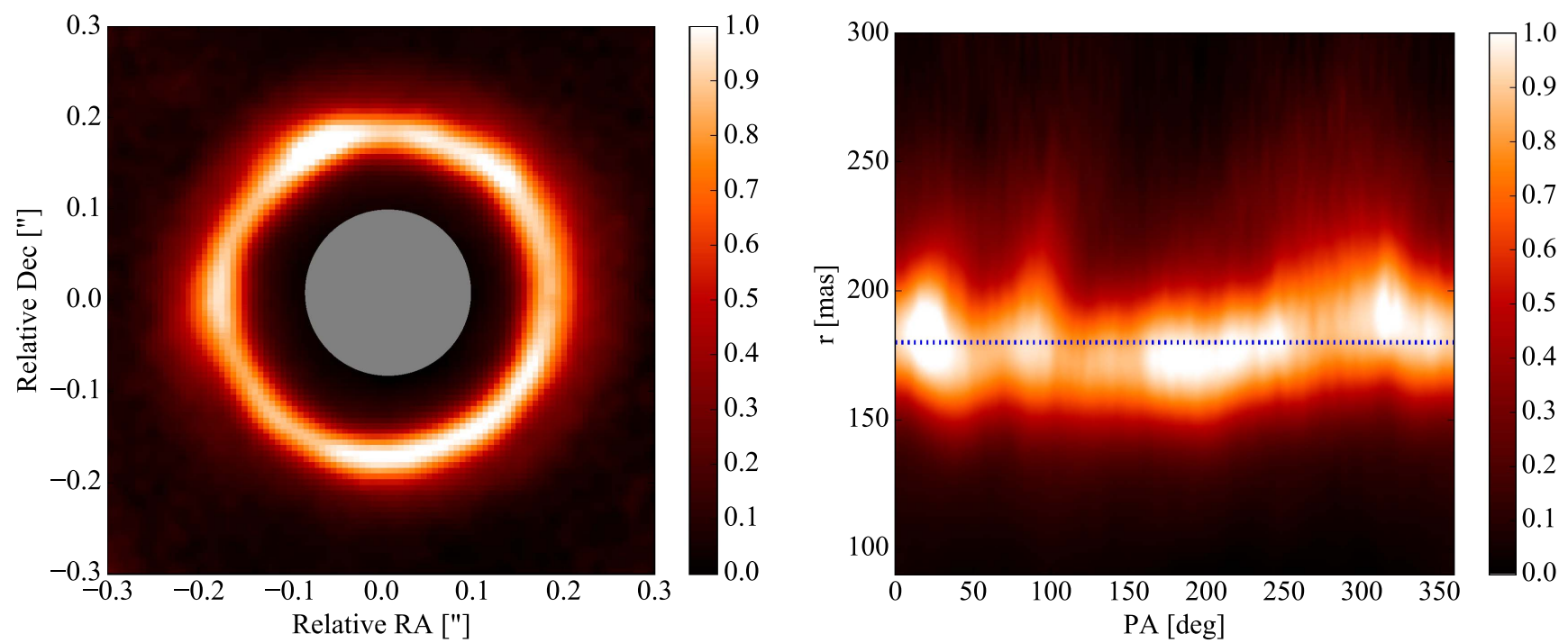

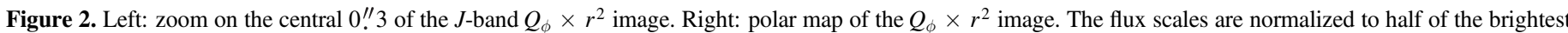
pixel along the ring. The horizontal dashed line indicates a radius of 0 ." 18 .

of the surface brightness, obtained after deprojection and azimuthally averaging the image (Figure 3, left).

(b) A ring (Ring \#1) peaking at 0".56 ( 66 au) with an apparent width of $\sim 0$ ! $^{\prime \prime} 16\left(\sim 19 \mathrm{au}\right.$, at PA $\left.\sim 100^{\circ}\right)$. This outer ring also shows some azimuthal brightness variation with a dip in scattered light along PA $\sim-15^{\circ}-30^{\circ}$. This is also detected in the $H$ - and $J$-band images of Momose et al. (2015) and Monnier et al. (2017).

(c) A wide off-center gap (Gap \#2), the width of which ranges from 0 !" $13(\sim 15 \mathrm{au})$ along $\mathrm{PA} \sim 100^{\circ}$ to 0 "! $24(\sim 28 \mathrm{au})$ along PA $\sim 200^{\circ}$. In the left panel of Figure 3, it is also evident that this gap is not empty of scattering material, with a lowest value of $1 \%-2 \%$ of the peak value at $0 . " 35$ ( $\sim 41 \mathrm{au})$. We note, however, that it could actually be emptier, with light from the adjacent rings inside and outside being convolved into the gap. An additional polar map of the full image showing the various gap widths against position angle is available in Appendix A (Figure 11).

(d) A resolved bright and narrow ring (Ring \#2), located at 0 .' 18 ( $\sim 21 \mathrm{au})$ with an apparent width of 40-50 mas ( $\sim 5-6 \mathrm{au})$. Its brightness varies azimuthally by up to $\sim 25 \%$, as evidenced by the zoom displayed in the left panel of Figure 2. The regions at PAs $\sim 23^{\circ}, 90^{\circ}, 200^{\circ}$, and $315^{\circ}$ are brighter than the regions at PAs $\sim 0^{\circ}, 60^{\circ}, 130^{\circ}$, and $275^{\circ}$. Figure 2 (right panel) shows the image in polar coordinates after deprojecting it with the inclination and position angle derived from the observed kinematic pattern and line profiles at mm wavelengths $\left(i \sim 13^{\circ}\right.$ and $\mathrm{PA} \sim 5^{\circ}$, respectively). One can see that the ring does not lie on a perfectly horizontal line (at a radius of 0 ". 18 in the plot). This suggests that the ring is intrinsically asymmetric or could be asymmetrically illuminated due to shadowing by the inner disk. The ring might also have a nonnegligible vertical extent, although this is rather unlikely due to the face-on disk configuration. A detailed analysis of the geometry of this inner ring based on optical SPHERE-Zurich IMaging POLarimeter (ZIMPOL; Thalmann et al. 2008; Schmid et al. 2012) data can be found in G.H.-M. Bertrang et al., (in preparation).

(e) A region with a deficit of scattered light (Gap \#3) outside of our IWA $(0$ ". 08$)$. This inner gap appears devoid of scatteredlight flux, but there is an unresolved inner disk with accretion as discussed in Grady et al. (2007) and Wagner et al. (2015).
Figure 3 (right panel) shows the azimuthal cuts along the two rings after deprojecting the $Q_{\phi}$ image and radially averaging over their apparent widths (between 0 !" 14 and 0 "! 22 and between 0 " 40 and 0 ". 65 , respectively). One can see that both the inner and outer rings present clear azimuthal variations. The outer disk appears brighter along PA $\sim 110^{\circ}-120^{\circ}$, i.e., close to the minor axis of the disk. To better characterize the rings and Gap \#2, we attempt to fit ellipses to the image. We follow the procedure described in detail in de Boer et al. (2016) and Ginski et al. (2016): we consider $10^{6}$ annuli for each feature and find the annulus for which the flux is maximized (for the rings) or minimized (for the gap). To reduce the number of free parameters, we fix the inclination and position angle of the ellipses to the values inferred from interferometry (Panic et al. 2008). Our best-fit result is shown in Appendix B, in Table 1. We give the offset of the ellipses from the star position, as well as the size of the major and minor axes for each fitted feature. Our error bars are estimated as the standard deviation of the best $1 \%$ fits (i.e., the $1 \%$ fits with the highest flux in the resulting aperture for the rings). All of the offsets that we measure are toward the northwest direction (as in Momose et al. 2015), which suggests that the southeast side of the disk is the near side of the disk. However, we note that the direction of the offsets is not exactly along the minor axis, which might indicate that these offsets do not only trace geometrical effects and that the disk could be eccentric.

The SPHERE/IRDIS $J$-band image is very similar to the $H$ and $J$-band images obtained by Momose et al. (2015) with Subaru/HiCIAO and by Monnier et al. (2017) with the Gemini Planet Imager (GPI) $3 \mathrm{yr}$ and $1 \mathrm{yr}$ before our observations, respectively. This suggests that the observed azimuthal asymmetries are not due to shadowing from the innermost disk. Dynamical structures in the inner disk would evolve significantly on timescales of years (cf. discussion in Section 5.6). The two rings in our image are approximately colocated with the two rings detected in the ALMA millimeter dust continuum (Fedele et al. 2017), as shown in Figures 4 and 8. More precisely, the peaks of the two rings at $\mathrm{mm}$ are slightly further out than in our SPHERE scattered-light data $(\sim 28$ and $\sim 70$ au versus $\sim 21$ and $\sim 66 \mathrm{au}$ ), consistent with current dusttrapping scenarios. Although Gap \#2 does not appear devoid 

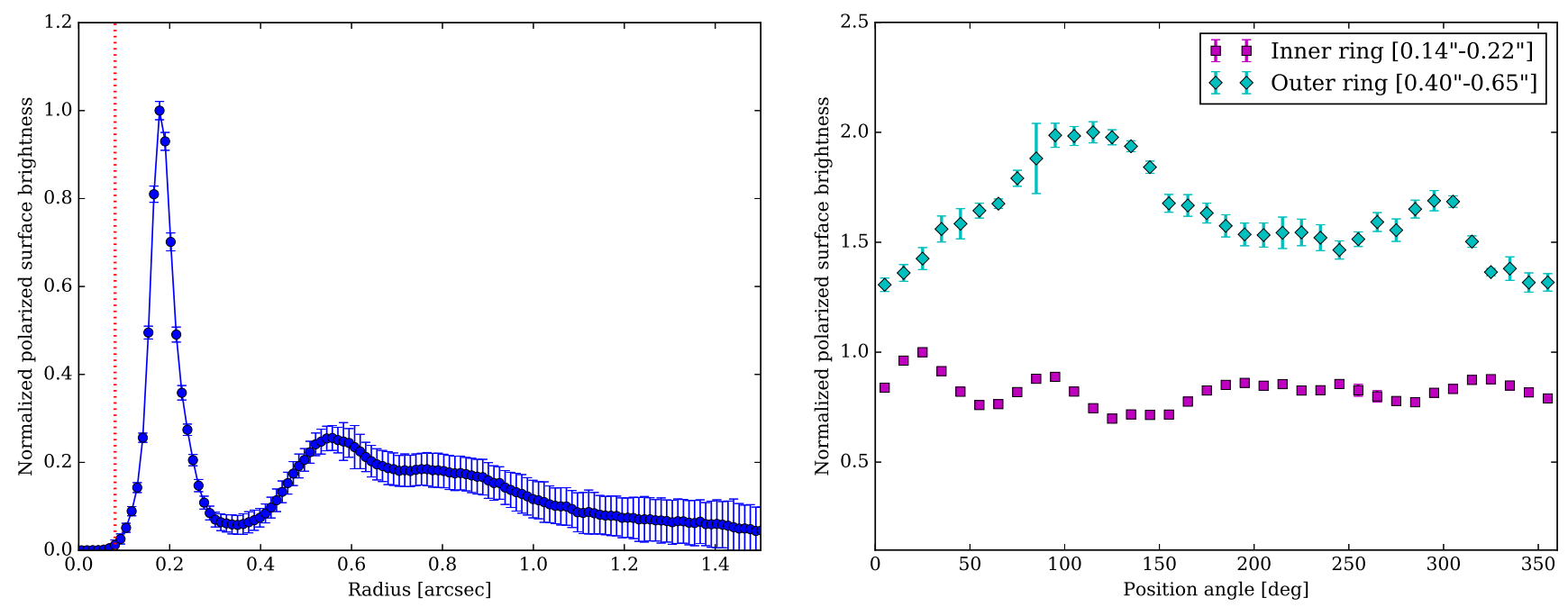

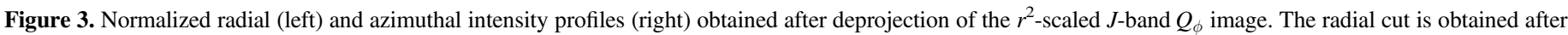

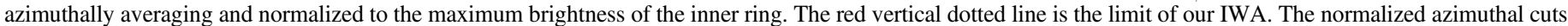

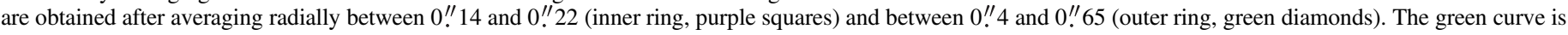
shifted vertically for clarity. The plotted error bars are the standard deviation in each bin in the $U_{\phi}$ image on a pixel basis.

of small dust grains, the ALMA image shows no continuum detection in both the inner (Gap \#3) and wide (Gap \#2) gaps. This indicates that dust particles, independently of their sizes, are filtered out in the inner gap but that the filtering mechanism at play in the outer gap affects small and large dust grains differently. The inner ring (Ring \#2) is also well detected in VLA 7 and $9 \mathrm{~mm}$ observations (Osorio et al. 2014; Macías et al. 2017), although at a slightly larger radius ( $\sim 25 \mathrm{au}$, compared to $\sim 21$ au in scattered light). Furthermore, Macías et al. (2017) also reported on the detection of a third gap at $\sim 85 \mathrm{au}$, consistent with the marginal detection in the SPHERE polarized intensity data.

\section{Disk Modeling}

We start our models by introducing planet-induced depressions in a uniform disk gas density profile to mimic the position and shape of the observed gaps. We present physical simulations, including dust evolution and trapping processes, to constrain the disk's dust distribution and investigate whether planet-disk interactions are responsible for the detected substructures. We take the approach of fixing as many parameter values as possible and do not attempt a best-fitting procedure. Because of the high parameter degeneracy when physical processes related to dust evolution are involved, we do not explore a large grid of these models. Our concept is complementary to the one presented by Monnier et al. (2017), who showed a parameterized model without connecting the gap and ring structures to a planetary origin or dust evolution. In their model, the scale height of an inner and outer disk region and the density scaling factor for the outer gap are determined via a fitting process.

\subsection{Model Set-ups}

In our models, we consider two spatially separated planets that are massive enough to open a gap in the gas surface density. The planet cores are assumed to be at fixed orbits and are not allowed to migrate. We note that we constrain the total number of planets to two, although multiple low-mass planets in close-by resonances could exist to cause the second, wide gap

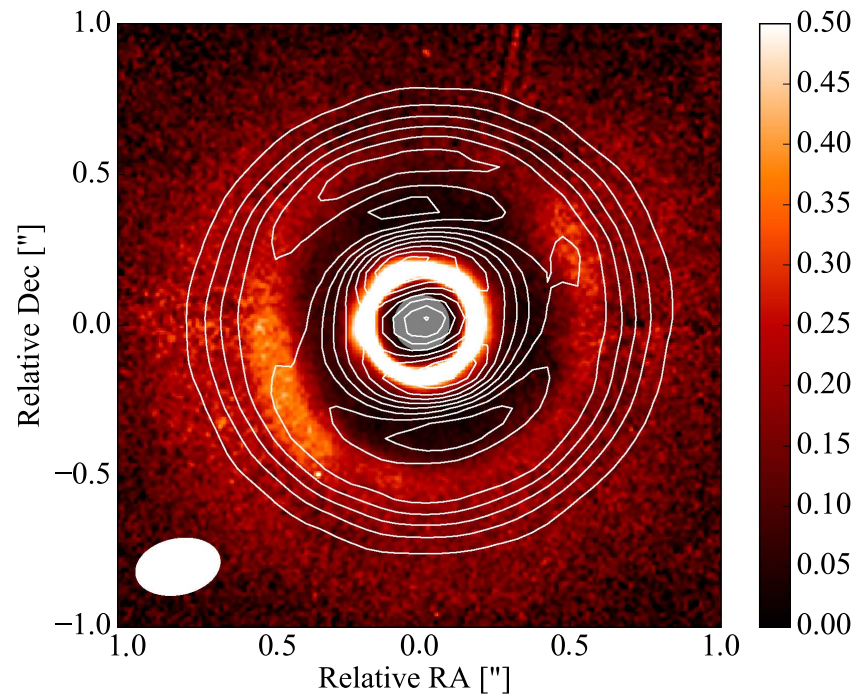

Figure 4. SPHERE/IRDIS $r^{2}$-scaled $J$-band $Q_{\phi}$ image overlaid with contours of the ALMA $1.3 \mathrm{~mm}$ continuum image (from Fedele et al. 2017). The white ellipse in the bottom left corner shows the ALMA beam with a size of 0 . $28 \times 0$ ". 18 . The $Q_{\phi}$ image is normalized in the same way as in the right panel of Figure 1, but the color scale is chosen such that the structures in the outer disk are enhanced and the inner ring is saturated.

(Gap \#2). The perturbed gas surface density profiles $\Sigma_{\mathrm{g}}$ depend on the planet masses and disk viscosity. To derive $\Sigma_{\mathrm{g}}$, we consider the analytical solution of Crida et al. (2006), in which the gravitational and pressure torques are assumed to be zero very close to the planet. For this reason, we implement a correction for the depth of the gap using the empirical relation from Fung et al. (2014). The resulting gas surface density distributions are used as inputs to model the dust evolution considering the dust dynamics, including the processes of coagulation, fragmentation, and erosion of dust particles (Birnstiel et al. 2010; Pinilla et al. 2015a). For the background surface density profile, we use an exponentially tapered power law with a power index of 1 and a tapered radius of six times the location of the inner planet. As the inner gap appears 
relatively devoid of scattering material and free from larger grains, we consider a planet-to-stellar mass ratio of $2 \times 10^{-3}$ $\left(3.5 M_{\text {Jup }}\right)$ for the inner planet, such that the gap is deep enough to lead to a filtration of particles of all sizes. For the second gap, filled with small particles, we consider the mass of the outer planet close to the mass estimate obtained in Osorio et al. (2014) in this region, and we choose 0.7 and $0.3 M_{\mathrm{Jup}}$, the latter being the minimum mass needed to open a gap in the gas surface density (and hence to have a pressure trap at the outer edge of the gap) under our assumptions. The locations of the planets are chosen according to the current SPHERE observations and are $r_{1}=14 \mathrm{au}$ and $r_{2}=53 \mathrm{au}$, such that the pressure maxima are close to the observed peaks of the mm emission. The companion masses considered in our simulations are compatible with the detection limits obtained in total intensity with IRDIS and the Integral Field Spectrograph (IFS) of SPHERE. These data will be presented in detail in the F100 SPHERE High-Contrast Imaging Survey for Exoplanets (SHINE) data analysis and detection performance paper (M. Langlois et al., in preparation). The disk temperature profile is a power law $\left(\sim r^{-0.5}\right.$; cf. Equation (25) in Birnstiel et al. 2010) such that at 1 au the temperature is $\sim 230 \mathrm{~K}$. We assume an $\alpha$ viscosity of $10^{-3}$ throughout the disk and note that this choice also influences the planet masses assumed as described above. Furthermore, we consider a disk mass of $5 \times 10^{-3} M_{\odot}$, which is consistent with the value range reported by Panić et al. (2008), and a disk radial extension from 1 to $300 \mathrm{au}$. The initial gas-to-dust ratio is 100 , and particles are initially $1 \mu \mathrm{m}$ in size. The model follows the evolution of 180 grain sizes (from $1 \mu \mathrm{m}$ to $2 \mathrm{~m}$ ) and calculates the dust density distribution at each radius for timescales from $10^{4}$ to $10^{7} \mathrm{yr}$. We do not consider the effect of ice lines on the dust dynamics.

To compute synthetic images, we consider the resulting dust distribution as input to the radiative transfer code RADMC-3D (Dullemond et al. 2012). From the vertically integrated dust density distribution, we derive the dust density for each grain size $\Sigma_{d}(r, a)$. From the temperature profile $T(r)$ used in the dust evolution, the pressure scale height $H_{p}(r)$ is determined. We take the approach in Pohl et al. (2016) and calculate the dust scale height for each grain size $a$ following Birnstiel et al. (2010) as

$$
H_{d}(r, a)=H_{p}(r) \times \min \left(1, \sqrt{\frac{\alpha}{\min (\mathrm{St}, 1 / 2)\left(1+\mathrm{St}^{2}\right)}}\right),
$$

where $\alpha$ is the turbulent viscosity and St is the Stokes number, a dimensionless parameter that indicates how well a dust grain is coupled to the gas. In the Epstein regime, valid for most regions of protoplanetary disks and where the molecular hydrogen mean free path is larger than 4/9 times the grain size, the Stokes number at the midplane can be written as

$$
\mathrm{St}=\frac{\rho_{s} a}{\Sigma_{\mathrm{g}}} \frac{\pi}{2}
$$

where $\rho_{\mathrm{s}}$ is the volume density of the dust grain, typically $\sim 1.2 \mathrm{~g} \mathrm{~cm}^{-3}$ according to the averaged values of the volume density for silicates. Dust grains with sizes corresponding to St $\sim 1$ are subject to the strongest gas drag and move fast to the regions of pressure maxima (Brauer et al. 2008). From the dust surface density and scale height, we compute the volume density profile for each grain size as

$$
\rho(R, \phi, z, a)=\frac{\Sigma_{d}(R, a)}{\sqrt{2 \pi} H_{d}(R, a)} \exp \left(-\frac{z^{2}}{2 H_{d}(R, a)^{2}}\right),
$$

where $R=r \sin (\theta)$ and $z=r \cos (\theta)$ are cylindrical coordinates and $\theta$ is the polar angle. The opacity calculation of each grain size bin takes into account porous spheres with a dust mixture composed of astronomical silicates (Draine 2003), carbonaceous material (Zubko et al. 1996), and water ice (Warren \& Brandt 2008). The fractional abundances of $7 \%$, $21 \%$, and $42 \%$ (amount of vacuum is $30 \%$ ) are adopted from Ricci et al. (2010). The temperature structure of each dust grain size is determined with a Monte Carlo radiative transfer simulation, and synthetic scattered-light images are computed including the full treatment of polarization. Mie theory is used to compute the Mueller matrix elements. These images are convolved by an elliptical Gaussian PSF $(0$." $034 \times 0$." 041$)$ chosen to mimic the angular resolution of our SPHERE observations, and each pixel is multiplied by the square of its distance to the star to compensate for the stellar illumination drop-off with distance. For the synthetic mm observations, we consider a beam size of 0 "! $3 \times 0$ "! 2 (Fedele et al. 2017).

For comparison reasons, we additionally perform simplified models by neglecting the self-consistent dust evolution-that is, the dust growth-and its dynamics. However, there are $\mathrm{mm}$ grains in these models, so significant evolution has taken place here as well. In this second approach, we consider the same initial gas density profile perturbed by the two giant planets and assume a fixed gas-to-dust ratio exploring the range from 50 to 100 , a simple approach typically used to compare scattered-light images with hydrodynamical simulations of planet-disk interaction (e.g., Dong \& Fung 2017). This approach is valid as long as the micron-sized particles are well coupled to the gas and no self-consistent dust settling is included. However, these simplified models are expected to differ from dust evolution models because several processes, such as growth and fragmentation, can change the dust distribution in the disk, which at the same time changes the dynamics, in particular when pressure maxima are present (cf. Figure 5). In these simplified models, an average opacity is used considering a power-law distribution for the grain size, where the number density follows $n(a) \propto a^{-3.5}$ with $a_{\min }=0.01 \mu \mathrm{m}$ and $a_{\max }=1 \mathrm{~mm}$. For all models, we consider the stellar parameters $T_{\mathrm{eff}, *}=8400 \mathrm{~K}$, $M_{*}=1.65 M_{\odot}$, and $R_{*}=1.5 R_{\odot}$, hence $10 L_{\odot} \quad($ Dunkin et al. 1997; Blondel \& Djie 2006; Fedele et al. 2017). The stellar luminosity adopted in Fedele et al. (2017) is based on the new distance estimate from Gaia $(d=117 \mathrm{pc})$. For the stellar spectrum, a Kurucz spectrum of a star with metallicity $[\mathrm{Fe} / \mathrm{H}]=$ 0 and a surface gravity of $\log g=4.5$ is taken into account (cf. Folsom et al. 2012).

In addition, as we do not know the shape of the innermost disk (masked by the coronagraph), and because the presence of a tiny amount of dust could alter the brightness signal close to the inner peak, we set the dust density to a floor value within $10 \mathrm{au}$ and apply a smooth Gaussian taper to create a rounded inner rim for the inner ring. This step is especially needed in the simplified models, because in the models with dust evolution included, most of the dust particles are filtered out and trapped at the outer edge of the gap opened by the innermost planet; 

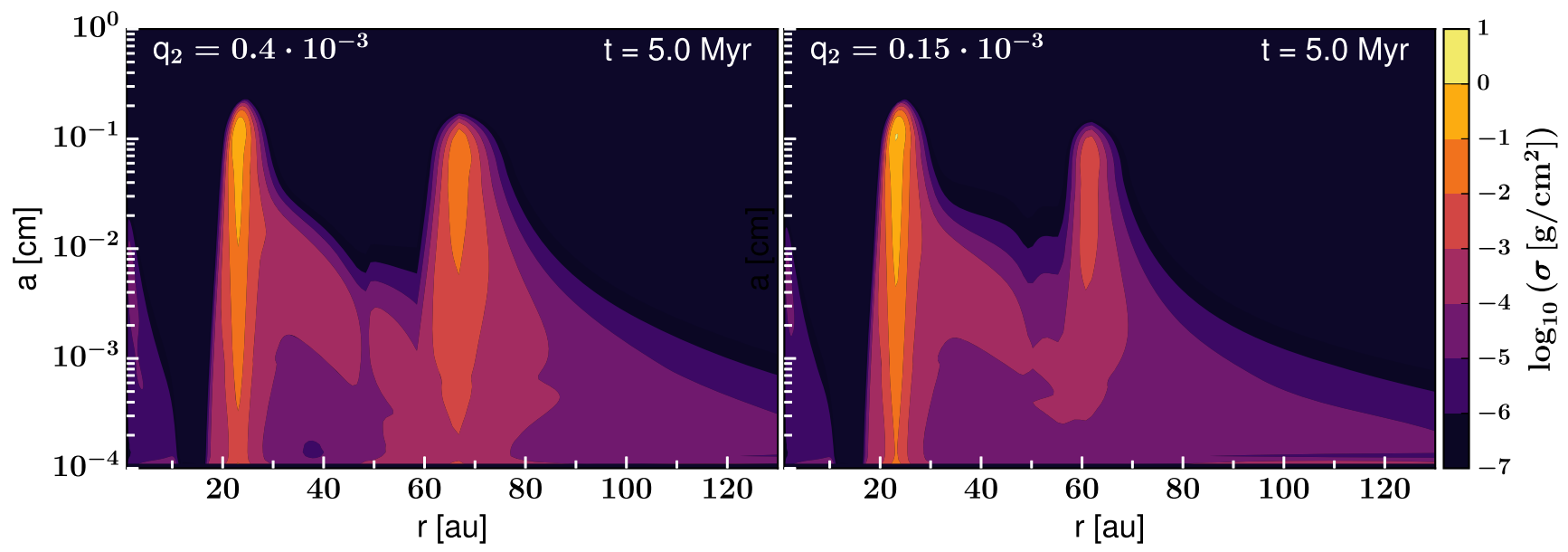

Figure 5. Vertically integrated dust density distribution after $5 \mathrm{Myr}$ of evolution, when two massive planets (left: 3.5 and $0.7 M_{\mathrm{Jup}}$; right: 3.5 and $0.3 M_{\mathrm{Jup}}$ ) are embedded in the disk at 14 and 53 au, respectively.

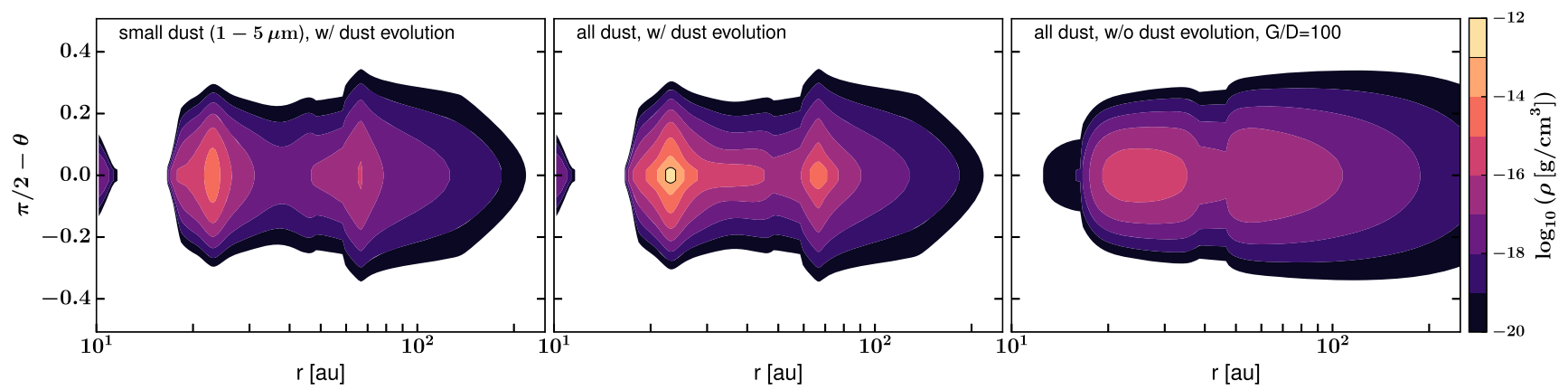

Figure 6. Vertical disk dust density structure assumed for the radiative transfer calculations following Equation (5) for an outer planet mass of $0.7 M_{\mathrm{Jup}}$. The cumulative density distribution for small dust grains only from 1 to $5 \mu \mathrm{m}$ (left), all dust grains (middle), and the simplified parametric approach (right) are shown. Note that the radial scale is logarithmic for a better visualization.

hence, in these models, the inner disk is almost empty of grains. However, we note that a tiny inner disk exists as presented in Lazareff et al. (2017).

\subsection{Results}

Figure 5 (left panel) shows the dust density distribution after $5 \mathrm{Myr}$ of evolution for planet masses of 3.5 and $0.7 M_{\text {Jup }}$ located at 14 and $53 \mathrm{au}$, respectively. A pressure bump is formed at the outer edge of each gap that acts as a particle trap and helps to reduce the radial drift. The higher the mass of the planet, the more efficient the trapping and higher the mm flux (Pinilla et al. 2012, 2015b). This trend is also seen in Figure 5 (right panel), where the second planet has a lower mass $\left(0.3 M_{\mathrm{Jup}}\right)$, leading to less efficient trapping there. Although the trapping of $\mathrm{mm}$ grains is effective, the values for the planet mass (at the considered disk turbulence) are chosen such that the small grains are not fully filtered out. Note that there is a degeneracy between the choice of disk mass, temperature, $\alpha$ turbulence, and planet mass; thus, we do not claim to infer mass limits for potential planets. The density contrast between the two rings also depends on whether the planets formed simultaneously or sequentially (Pinilla et al. 2015a) and whether they migrate. Applying Equation (5) to the dust density distributions results in the vertical density structure illustrated in Figures 6 (left and middle panels) and 12 (Appendix C). While the small grains are distributed radially over the disk extension and all the way up to the disk surface layers according to their dust scale height, the large grains are concentrated at the pressure bump regions close to the midplane. For comparison, Figure 6 (right panel) shows the vertical density structure of our simplified approach, where no grain growth model is involved and a fixed gas-to-dust ratio of 100 is considered. In this case, a larger amount of dust is still present within the two gap regions and in the outer disk because the dust radial drift is neglected.

\subsubsection{Scattered Light}

Figure 7 shows the radial surface brightness profiles of the $Q_{\phi} \times r^{2}$ model images compared to the observational radial profile (also shown in Figure 3). All profiles are normalized to the peak flux of the inner ring (Ring \#2). The brightness of this ring in scattered light is highest because of the geometry of the disk scattering surface. The incidence angle of stellar radiation is steepest here so that the disk receives and scatters the most light per unit surface area. This is due to density effects given the large amount of dust there and the temperature profile. The curves in the left panel are based on our dust evolution modeling approach. The solid green curve representing the model with a higher outer planet mass well reproduces the two main ring locations observed with SPHERE, but there is a discrepancy for the brightness contrast between the rings. Although the overall width of Gap \#2 matches the observations, a gap much deeper and with sharper edges than observed is produced by our model. Reducing the mass of the outer 

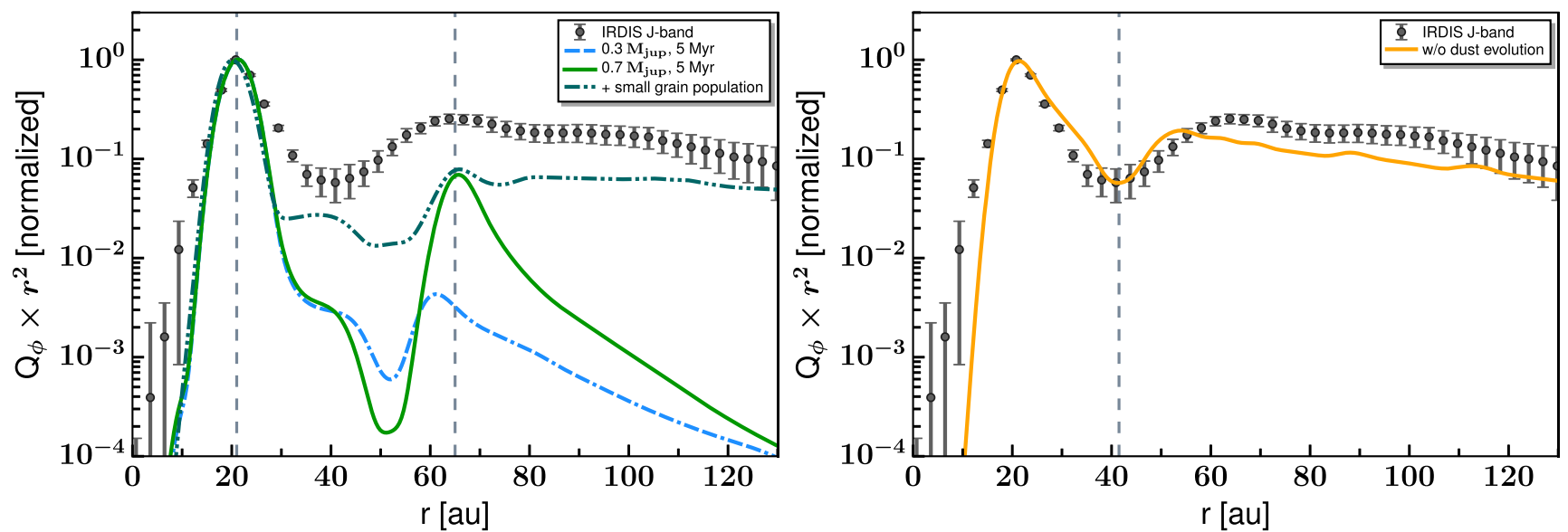

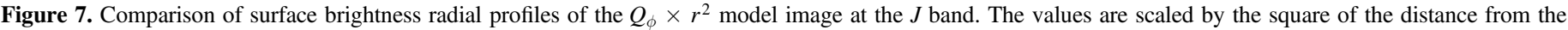

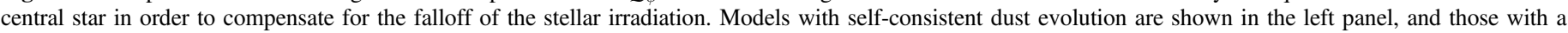

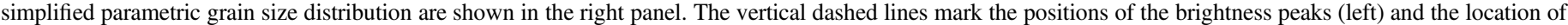
Gap \#2 (right).

planet helps to create a slightly shallower outer gap edge (dashdotted blue curve). However, in this case, the amount of dust that is trapped in the outer disk is lower, and, consequently, the brightness of Ring \#1 decreases. In addition, the peak of this outer ring moves slightly inward when the planet mass is reduced, differing from the observations. In both cases, the outer disk in our models appears too faint in scattered light, as all dust grains originally located in the outer disk have already grown and drifted inward, even after an evolutionary time of $0.5 \mathrm{Myr}$ (see Appendix D, Figure 13 and dashed olive curve in Figure 14).

The orange line in the right panel illustrates a good match for our simplified approach that ignores dust growth and fragmentation processes and assumes a power law for the dust size distribution. The gap location between the two main rings can be reproduced well when moving the second planet position further in from 53 to $42 \mathrm{au}$. The reason why the planet position needs to be different between the dust evolution models and the simplified approach is as follows. The gap in the small grains is similar in shape to that of the gas surface density, since the small grains are well coupled to the gas. Contrarily, in the dust evolution models, there is a dominating peak of emission at the pressure maximum, which is further out from the outer edge of the gas gap and where small grains are continuously reproduced by fragmentation due to turbulent motions (Pinilla et al. 2012). The shallowness of the gap is a better match compared to the dust evolution approach. However, a simultaneous fit of gap depth and ring positions is not possible either. This trend is also seen in the HD 169142 model-fitting results by Monnier et al. (2017), where either the gap depth or the outer ring position is off compared to the GPI $J$ - and $H$-band profiles.

Mixed midplane-surface dust models. At this point of the analysis, it seems that the model without dust evolution does a significantly better job reproducing the SPHERE scattered-light observations, which is, however, not the case for the mm dust continuum, as demonstrated later in Section 4.2.2. One has to keep in mind that dust evolution assumptions are developed for the disk midplane, where dust growth is quite efficient due to the high densities. Since the dust evolution models are only $1 \mathrm{D}$, the vertical disk structure chosen influences the situation at larger height. More precisely, the coagulation equation itself is not only calculated in the midplane, but it averages the processes with presumed weights over the vertical structure. Then, it assumes that the size distribution at a given location develops as a whole, followed by a redistribution of the grains. There is also a reservoir of small grains produced that are going through the growth and fragmentation cycle. It might be that the vertical exchange in the dust evolution is not working properly and that there are small grains at the disk surface that do not grow quickly at high altitude where the densities are lower. If this population at the top layer is indeed isolated, its coagulation compared to the midplane situation will also be on a different timescale. Moreover, charging effects could play a role for dust evolution processes at the disk surface, which would keep the particles very small.

Hence, as a test, we introduce a new population of small grains $(0.01-0.5 \mu \mathrm{m})$ that follow the initial gas density distribution of the dust evolution model with a mass fraction of $8 \times 10^{-4} \Sigma_{\text {disk,gas. }}$ The mass in the other size bins is reduced correspondingly to keep the same dust mass as that for the original dust evolution simulation. This model is displayed in Figure 7 with the dash-dotted dark green line. It helps to decrease the gap depth and increase the scattered light in the outer disk.

We note that our models that do not contain any dust inside $10 \mathrm{au}$ provide a good match to the SED for wavelengths longer than $10 \mu \mathrm{m}$ that trace the outer disk. However, the addition of a small inner belt between $\sim 0.05$ and 0.09 au allows us to reproduce the NIR excess as well. To not be seen in our scattered-light model predictions, any dusty material in the inner disk must be confined within $\sim 0.09$ au.

\subsubsection{Millimeter Dust Continuum Emission}

Figure 8 shows synthetic mm continuum images at $1.3 \mathrm{~mm}$ for our two representative models (solid lines in Figure 7) alongside the ALMA data from Fedele et al. (2017). The left panel considers our self-consistent dust growth model. This results in an inner dust cavity, an inner ring between $\sim 15$ and $35 \mathrm{au}(0 . " 13$ and $0 . " 3)$, and an outer ring between $\sim 55$ and $80 \mathrm{au}(0$ " 47 and 0 " 68$)$, with a gap in between. Both the inner cavity and the gap are depleted in mm-sized dust particles. Our model is qualitatively consistent with the ALMA dust continuum image showing rings at $\sim 20-35$ au and 

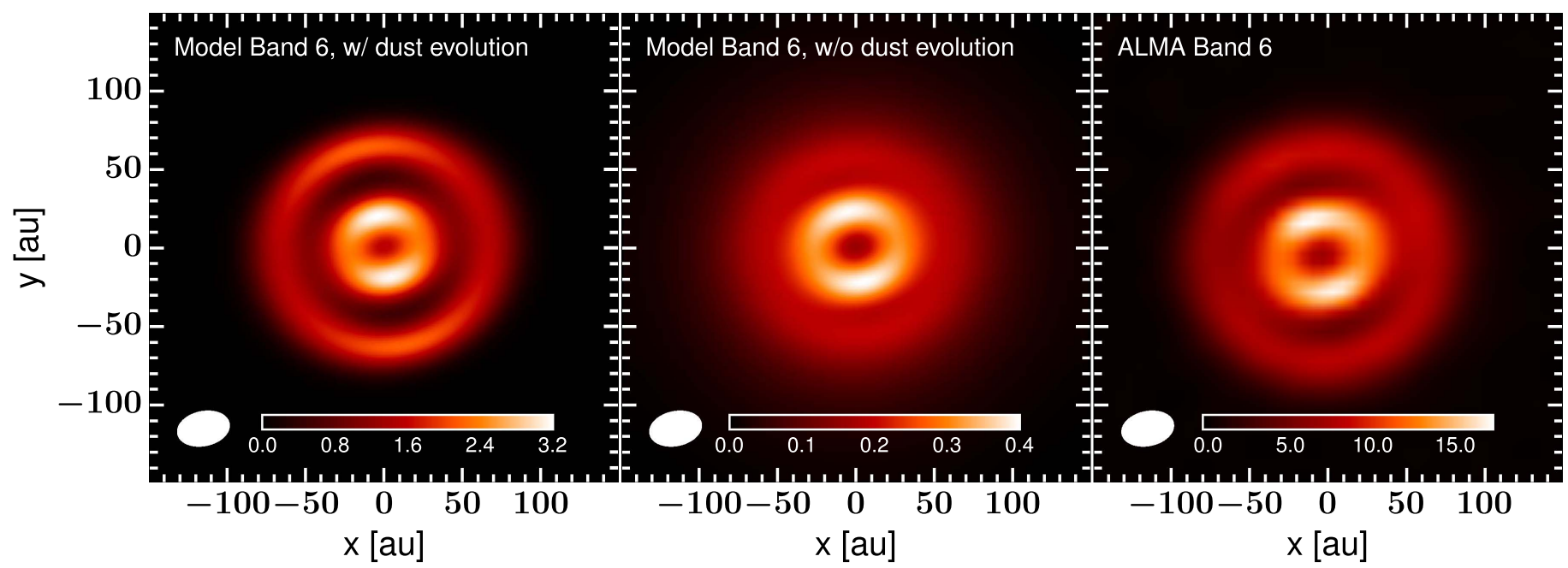

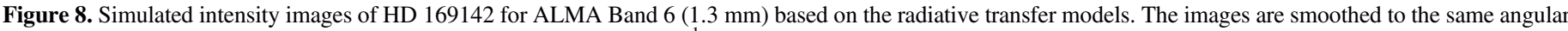
resolution as in Fedele et al. (2017). The intensity units are mJy beam ${ }^{-1}$.

56-83 au (0". 48-0".64). This agreement supports the view that the efficient dust-trapping scenario by means of the two giant planets may be at work in HD 169142. The relatively sharp outer edge of the continuum map gives further evidence of large dust grains radially drifting inward (cf. Birnstiel \& Andrews 2014; Facchini et al. 2017). For completeness, the synthetic image for our simplified fixed gas-to-dust ratio model without dust evolution treatment is also shown. The clear depletion of dust particles within the gap region is not seen in this case. Furthermore, the outer ring is more extended, and both the inner and outer edges are less well defined, leading to a fuzzier overall disk structure. We note that the flux is underpredicted in both model scenarios compared to the actual ALMA measurement.

\section{Discussion}

\subsection{Fragmentation and Trapping Efficiency}

The main reason for the discrepancy between the dust evolution models and the simplified approach is the fact that, in the dust evolution models, micron-sized particles efficiently grow to larger sizes already at early times of evolution. The growth changes their coupling to the gas (i.e., their Stokes number) and hence their dynamics (e.g., their drift velocities increase when they grow). In these models, small grains are continuously reproduced thanks to destructive collisions that can occur because of turbulent motions and radial drift. In the particular case of two planets embedded in the disk presented in this study, the radial drift is reduced at the pressure bumps, and fragmentation occurs due to turbulence that replenishes these regions with small grains. These small grains are more affected by turbulent motions and more difficult to trap, and thus they can be dragged along with the gas. For this reason, a small amount of micron-sized particles can still flow through the gap (cf. Figure 5). This amount of small grains is, however, not enough to reproduce the observed surface brightness profile inside the gap (as it is in the case of a constant gas-to-dust ratio). For sub-micron grains, this amount would be significantly higher, and these smaller grains scatter more efficiently in our direction, too. A possible solution for this discrepancy is to make fragmentation more efficient, for example, by increasing the turbulent motions of the grains, that is, increasing the $\alpha$ viscosity. However, when $\alpha$ increases, a more massive planet is needed to open a gap (Crida et al. 2006), which can lead to a new discrepancy with the gap width. Moreover, the higher the turbulent motions, the more difficult it is to trap the mm-sized particles, because of the high dust diffusion that allows particles to escape from pressure bumps (de Juan Ovelar et al. 2016). As an alternative, in order to have less growth and more fragmentation, the maximum fragmentation velocity threshold of the particles can be decreased, which mainly depends on the grain composition and its structure. Nonetheless, while having more fragmentation might help to increase the surface brightness inside the gap, this can also lead to less dust trapping, which can create differences with the current $\mathrm{mm}$ observations. Note that decreasing the initial minimum grain size in our current dust evolution models would not help us have a better match with observations, because small grains quickly grow regardless of their initial size.

\subsection{Dust Evolution as a Function of $z$}

Following the analysis in Section 4.2.1, it becomes clear that the assumptions in current 1D dust evolution models are tuned for coagulation processes happening in the disk midplane. The evolution of gas and dust is modeled in a vertically integrated way assuming a steady-state disk model, although there might actually be a strong dependency on the vertical disk height. Hence, our treatment of vertical exchange and vertical settling might also be inaccurate. For instance, if there is indeed a population of small grains isolated at the top layer, this would suggest very weak turbulence. This provokes quite efficient settling, even for small grains. What might work is a population of small, charged grains that is kept from settling by, for example, magnetic fields. Hence, these small grains would be unaffected by efficient dust growth and could be permanently present at the disk surface. As a consequence, scattered-light detections at optical and NIR wavelengths would not be affected by significant dust growth. Contrarily, the surface layers might have higher turbulence, which is expected because they are hotter and highly ionized (e.g., Dzyurkevich et al. 2013). However, with only higher turbulence, grains are also mixed downward and get into contact with the lower turbulence regions deeper in the disk, where they settle and take part in the coagulation there. Thus, a locally higher turbulence is not a way to isolate grains; it is a way to move 
them faster. Consequently, it could be that the gas velocities at very high altitude are such that fragmentation also works in a thin surface layer to locally replenish the reservoir of even submicron grains.

\subsection{Mass of Gap-opening Planets}

As shown in Section 4.2, planets with masses of 3.5 and $0.7 M_{\text {Jup }}$ located at 14 and 53 au are needed in our dust evolution models in order to create effective pressure bumps that trap particles at the location of the rings seen in scattered light. Note that these values are compatible with the mass detection limits derived from contrast curves in total intensity SPHERE IRDIS and IFS data. The minimum planet mass limit in our model is chosen such that the planet perturbs the gas profile and efficient trapping can be generated $\left(M_{\mathrm{p}} \gtrsim 0.3 M_{\text {jup }}\right)$. While a planet mass of $0.3 M_{\text {Jup }}$ is too low to clear the full extent of Gap \#2, the $0.7 M_{\text {Jup }}$ planet is able to reproduce the gap width. The presence of multiple planets below this mass whose gaps overlap is an alternative possibility (e.g., Dodson-Robinson \& Salyk 2011). Numerical studies have shown that less massive planets do not open a gap in the gas but effectively open a gap in the dust (Paardekooper \& Mellema 2004, 2006; Picogna \& Kley 2015; Dipierro et al. 2016; Rosotti et al. 2016; Dipierro \& Laibe 2017). The gas azimuthal velocities can be perturbed such that the drift velocities of the particles are reduced, leading to a traffic-jam effect without creating local pressure maxima. In addition to the gas viscous forces, Dipierro et al. (2016) and Dipierro \& Laibe (2017) also included the contribution from the tides of an embedded planet and showed that a low-mass planet can open a gap in the dust only if the tidal torque exceeds the drag torque outside the planetary orbit. In this scenario, a shallow gap can be carved out, but it is rather unlikely that this effect can create the strong rings in the distribution of small and large grains in HD 169142. It should be tested whether a combination of pressure bumps, self-consistent dust evolution, and the consideration of disk-planet tidal interactions can lead to a coherent picture for the gap and ring appearances.

Pioneering studies from Kanagawa et al. (2015), Rosotti et al. (2016), and Dong \& Fung (2017) look at the inverse problem, meaning to derive planet masses from observed gap profiles. For this method, a number of assumptions about the disk structure and dynamics are made when simulating the gap shape and deriving the correlation with planet mass. The mass of the putative second planet in our HD 169142 model is consistent with the numerical analysis by Dong \& Fung (2017), who derived disk and planet properties based on the morphology of gaps in NIR scattered-light images. They estimated a mass between 0.2 and $2.1 M_{\text {Jup }}$ for an $\alpha$ viscosity varying from $10^{-4}$ to $10^{-2}$. Kanagawa et al. (2015) suggested a mass $\gtrsim 0.4 M_{\text {Jup }}$ by measuring the gap depth in VLA $7 \mathrm{~mm}$ data. Although this is principally consistent with the other estimates, a measurement based on mm data only is complicated due to dust/gas coupling effects (Rosotti et al. 2016). This makes an exact definition of the gap width difficult, and its value depends on the disk lifetime. As discussed in Rosotti et al. (2016), a more robust indicator of the planet mass from (sub-)mm images is the location of the bright ring tracing the gas pressure maximum. This is the reason why we intend to reproduce the ring positions rather than the gap locations with the modeling approach in this paper. The inclusion of dust growth and fragmentation processes would certainly change the conclusions from Rosotti et al. (2016) and Dong \& Fung (2017) as dust evolution dynamics affects the gap depth, the slope of the gap edges, and the position of the rings and their contrast.

\subsection{Dust Evolution Timescale}

All of our results based on dust evolution modeling consider a dust evolutionary timescale of $5 \mathrm{Myr}$ and that the giant planets embedded were formed simultaneously. The disk and planet age can affect the appearance of the radial profiles in polarized intensity at the NIR (see Figure 14 in the Appendix D) and in total intensity at $\mathrm{mm}$ wavelengths. On the one hand, the outer ring (Ring \#1) becomes narrower at longer times of evolution, which produces a rather sharp outer disk edge and shifts it toward smaller radii. This in turns lowers the brightness signal in the outer disk. While this is consistent with the $\mathrm{mm}$ data, the amount of small dust particles decreases with time, and the NIR observations cannot be reproduced. If longer times of evolution are taken ( $\sim 10 \mathrm{Myr}$, which is consistent with the revised age of the system), there would be a higher discrepancy between the dust evolution models and the NIR observations unless additional trapping mechanisms play a role all across the disk. In contrast, at very early timescales of $0.1-0.5 \mathrm{Myr}$, when all grains are not yet at the pressure maxima, the wide gap (Gap \#2) remains shallower. On the other hand, analogous to the sequential planet formation scenario presented in Pinilla et al. (2015b), it could be possible that the outer planet forms earlier than the inner planet (or vice versa). This can affect the amount of dust in both traps and adjust the contrast between the two rings. However, we do not have any constraint on whether the two planets have been forming at the same time or consecutively. Together with the uncertainty when the putative planets have been forming at all, this means that the dust evolution after 5 Myr could still be a good proxy for the situation in the HD 169142 system.

\subsection{Gaps and Rings in the Context of Ice Lines}

Although observational signposts of embedded planets are the most widely used explanation to interpret ring structures in disks, the relation to ice lines of various materials is another possible scenario (Zhang et al. 2015; Okuzumi et al. 2016). Ice lines of different volatile species can significantly affect the dynamics of dust evolution processes including growth and fragmentation, which in turn has an effect on the observational appearance of rings and gaps at different wavelengths (Pinilla et al. 2017). The freeze-out temperatures of the main volatiles, such as water $\left(\mathrm{H}_{2} \mathrm{O}\right)$, ammonia $\left(\mathrm{NH}_{3}\right)$, carbon dioxide $\left(\mathrm{CO}_{2}\right)$, and carbon monoxide (CO), are estimated to have average values of $\sim 142, \sim 80, \sim 66$, and $\sim 26 \mathrm{~K}$, respectively (Zhang et al. 2015).

Our radiative transfer models show that the midplane temperatures at the inner ring position are such that the $\mathrm{H}_{2} \mathrm{O}$ and $\mathrm{NH}_{3} / \mathrm{CO}_{2}$ ice lines are located close to the inner and outer edges of this ring, respectively, at $\mathrm{mm}$ emission. It is recognizable that the gap at scattered light lies between the ice lines of $\mathrm{H}_{2} \mathrm{O}$ and $\mathrm{CO}_{2}$ when comparing the surface layer temperatures with the volatile freeze-out temperatures. Thus, the $\mathrm{H}_{2} \mathrm{O}, \mathrm{NH}_{3}$, and $\mathrm{CO}_{2}$ ice lines nearly coincide with the scattered-light ring positions. Furthermore, the $\mathrm{CO}$ ice line at the midplane is located at $\sim 110 \mathrm{au}$, which is close to the outermost gap at $\sim 85 \mathrm{au}$, consistent with $\mathrm{DCO}^{+}(3-2)$ and $\mathrm{C}^{18} \mathrm{O}(2-1)$ ALMA observations presented in Macías et al. (2017). In Figure 9, there is an uncertainty for the specific location of these ice lines that depends on the freezing temperatures that we assume, and, for the CO ice line, it could be between $\sim 95$ and 145 au. The current observations suggest that 


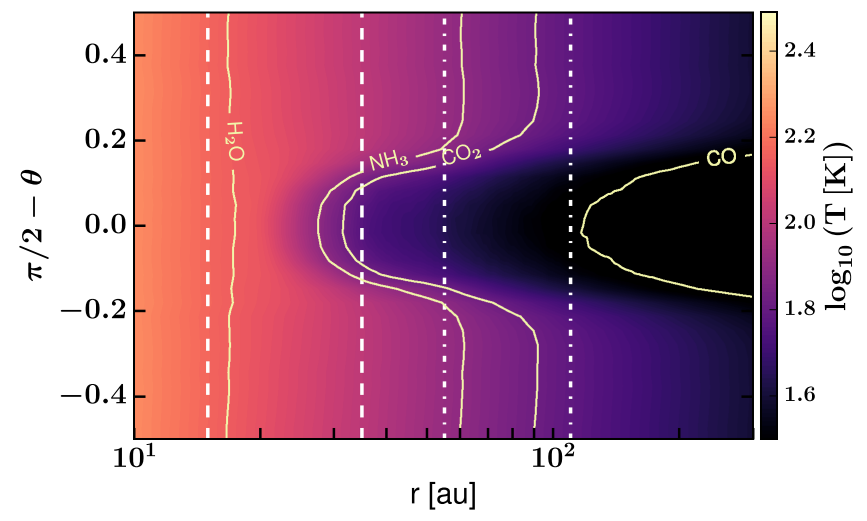

Figure 9. Temperature map as a function of radius and polar angle in spherical coordinates for the simplified model. The ice lines for $\mathrm{H}_{2} \mathrm{O}, \mathrm{NH}_{3}, \mathrm{CO}_{2}$, and $\mathrm{CO}$ are indicated with yellow contours. The vertical dashed lines illustrate the edges of the two bright rings in mm dust emission.

the accumulation of large dust grains close to the $\mathrm{CO}$ ice line is a possible mechanism to explain the origin of this outermost gap.

\subsection{Shadowing Effects and Time Variability}

We note that because of the modeling procedure (analytical gas profile coupled with 1D dust evolution), the observational signatures presented in this paper are always azimuthally symmetric. This is, for example, not necessarily true for a massive enough planet for which an eccentric gap and vortex formation at its edge are expected (e.g., Ataiee et al. 2013). As mentioned in Section 2, there are significant asymmetries along both the inner and outer ring regions in polarized intensity. It is noticeable that the maximum polarization of the outer ring is along the minor axis. This is opposite to several other disks showing a brighter polarized intensity along the major axis, expected due to the polarization efficiency being highest for $90^{\circ}$ scattering in the Rayleigh and Mie scattering regime. A significant scattering angle effect is also not expected at the low inclination of HD 169142. Momose et al. (2015) invoked corrugations of the scattering surface in the outer region as a possible origin. Alternatively, asymmetries in the outer disk emission can be caused by shadowing of the inner disk region. For HD 169142, there is a slightly inclined inner disk at sub-au distance $\left(i=21^{\circ}, \mathrm{PA}=100^{\circ}-130^{\circ}\right.$; Lazareff et al. 2017) that is known to be variable and might contain an extended dust envelope, as suggested by Wagner et al. (2015). Azimuthal brightness variations in the inner ring of the scattered light could be caused, for example, by perturbations by a protoplanet, optical depth variations through the suggested dust envelope, accretion flows, or turbulence in the inner disk. The local brightness enhancements along the innermost scattered-light ring at different time epochs are discussed in Ligi et al. (2017). The azimuthal inhomogeneities in the inner ring (Ring \#2) could in turn cause radial shadowing on Ring \#1 and the remaining outer disk. The pace of variations in the illumination pattern of the outer disk depends on the precession timescale of the inner disk material. Given that there is no apparent difference in the rings' brightness asymmetries in the three observational data sets in polarized intensity (Subaru/HiCIAO: Momose et al. 2015; Gemini South/GPI: Monnier et al. 2017; VLT/SPHERE: this paper) that span a time period of $3 \mathrm{yr}$, the shadowing scenario for the outer disk seems rather unlikely. However, it cannot be ruled out either, as the precession timescale for the inner disk, for example, in the context of a hypothetical star-companion system, can be rather long (several hundred to thousand years).

\section{Conclusions}

In this paper, we present scattered-light observations of the protoplanetary disk around the Herbig Ae star HD 169142 obtained with the VLT/SPHERE at the $J$ band and compare our results with recent ALMA data of this target. Together with TW Hya, HD 163296, and HD 97048, it is one of a handful of disks around young stars that have been observed at very high angular resolution at NIR and mm wavelengths, where in each case both images show similar substructures even if their scales differ but also show different morphologies. For HD 169142, we confirm the previous detection of two ringlike features separated by a wide gap and an additional inner gap and report on the marginal detection of a third gap in the outer disk, as well as azimuthal brightness variations along both rings. We present azimuthally symmetric radiative transfer models based on planet-disk interaction processes that account for the main observational features and discuss the influence of dust evolution and particle trapping on the gap and ring properties. We place our findings in the context of planet masses inferred from the gap-opening process and ice-line chemistry. Our measurements and modeling results suggest the following:

1. The location and width of the gap, as well as the peak positions in polarized scattered light, of HD 169142 are reproduced with our model based on dust evolution processes when two giant planets of 3.5 and $0.7 M_{\text {Jup }}$ are embedded in the disk. The observed gap, however, possesses a shallower outer flank than expected for planet-disk interaction signatures. There is also a significant discrepancy in the gap depth, as micron-sized particles rapidly grow in the presence of pressure bumps. Small grains distributed all over the disk wherever there is gas, as in our simplified approach, decreases the gap depth such that there is good agreement with the observed shape. This also helps to increase the scattered-light flux in the outer disk. A more efficient fragmentation by increasing the turbulent motion of dust particles or to adjust the fragmentation velocities could help to overcome this deficit in small grains. Including the contribution from the tides of an embedded planet can lead to a shallower dust gap, in case the planet hypothesis is correct at all.

2. The assumptions in current dust evolution models are tuned for the disk midplane, and the vertical exchange does not work properly; thus, the coagulation timescale might be different at higher disk altitudes. A population of small (sub)micron-sized grains might exist in the upper surface layers that is unaffected by quick growth due to lower densities and different turbulence there. Thus, the vertical disk structure and its consequences for dust evolution processes also have a significant role in interpreting scattered-light images.

3. In order to obtain a consistent picture with the $\mathrm{mm}$ observations, the accumulation of large grains in the dust trap of a pressure bump is needed. This generates the bright emission rings and the sharp outer disk edge as detected in the mm continuum image of HD 169142. A simplified parameterized dust size distribution is not able to reproduce the high dust depletion factor required.

4. A scenario with a grain size-dependent gap opening that still allows a perturbation in the radial pressure gradient is 
required. We emphasize that inferring the mass of gapopening planets from simplified models is degenerate and depends on the choice of disk mass, temperature, and $\alpha$ turbulence. Constraining planet masses becomes even more uncertain when including more physical processes that are expected to occur in protoplanetary disks, such as grain growth, fragmentation, and vertical disk instabilities.

5. Observing the total amount of gas and using different techniques that allow us to get better constraints on the grains sizes in disks, such as mm-wave dust polarization (Kataoka et al. 2015, 2016; Pohl et al. 2016; Yang et al. 2016), may allow us to further explain the origin of the gaps and derive the properties of potential embedded planets.

Eventually, 2D dust evolution models are needed in order to have a self-consistent treatment of radial transport and vertical settling and consider turbulence changes across the vertical direction of the disk. In principle, we can start to use multiwavelength analyses, such as the one presented in this paper, to provide feedback on the model assumptions and calibrate our understanding of microphysical dust processes (sticking, fragmentation, compact versus fluffy grains, etc.).

We acknowledge the ESO Paranal staff for their support during the observations. We thank J. Monnier for sharing his data. We are thankful to L. Pérez, A. Garufi, and G. Bertrang for the useful discussions about this project. A.P. is supported by the ReimarLüst Fellowship of the Max Planck Society and a member of the International Max Planck Research School for Astronomy and Cosmic Physics at Heidelberg University, IMPRS-HD, Germany. We acknowledge financial support from the Programme National de Planétologie (PNP) and the Programme National de Physique Stellaire (PNPS) of CNRS-INSU. This work has also been supported by a grant from the French Labex OSUG@2020 (Investissements d'avenir-ANR10 LABX56). M.B., F.M., and G.P. acknowledge funding from ANR of France under contract number ANR-16-CE31-0013 (Planet Forming Disks). P.P. acknowledges support by NASA through Hubble Fellowship grant HST-HF2-51380.001-A awarded by the Space Telescope Science Institute, which is operated by the Association of Universities for Research in Astronomy, Inc., for NASA, under contract NAS 5-26555. H.A., M.M., and S.P.Q. acknowledge support from the Millennium Science Initiative (Chilean Ministry of Economy) through grant $\mathrm{RC} 130007$ and further financial support by FONDECYT, grant 3150643, as well as financial support within the framework of the National Centre for Competence in Research PlanetS supported by the Swiss National Science Foundation. T.B. acknowledges funding from the European Research Council (ERC) under the European Unions Horizon 2020 research and innovation program under grant agreement No. 714769. D.F. acknowledges support from the Italian Ministry of Education, Universities and Research project SIR (RBSI14ZRHR). Q.K. acknowledges funding from STFC via the Institute of Astronomy, Cambridge Consolidated Grant. SPHERE is an instrument designed and built by a consortium consisting of IPAG (Grenoble, France), MPIA (Heidelberg, Germany), LAM (Marseille, France), LESIA (Paris, France), Laboratoire Lagrange (Nice, France), INAF-Osservatorio di Padova (Italy), Observatoire astronomique de l'Université de Genève (Switzerland), ETH Zurich (Switzerland), NOVA (Netherlands), ONERA (France), and ASTRON (Netherlands) in collaboration with ESO. SPHERE was funded by ESO, with additional contributions from CNRS (France), MPIA (Germany), INAF (Italy), FINES (Switzerland), and NOVA (Netherlands). SPHERE also received funding from the European Commission Sixth and Seventh Framework Programmes as part of the Optical Infrared Coordination Network for Astronomy (OPTICON) under grant number RII3-Ct-2004-001566 for FP6 (2004-2008), grant number 226604 for FP7 (2009-2012), and grant number 312430 for FP7 (2013-2016). This work has made use of data from the European Space Agency (ESA) mission Gaia (http://www. cosmos.esa.int/gaia), processed by the Gaia Data Processing and Analysis Consortium (DPAC; http://www.cosmos.esa.int/web/ gaia/dpac/consortium). Funding for the DPAC has been provided by national institutions, in particular the institutions participating in the Gaia Multilateral Agreement.

\section{Appendix A $U_{\phi}$ Image and Polar Mapping}

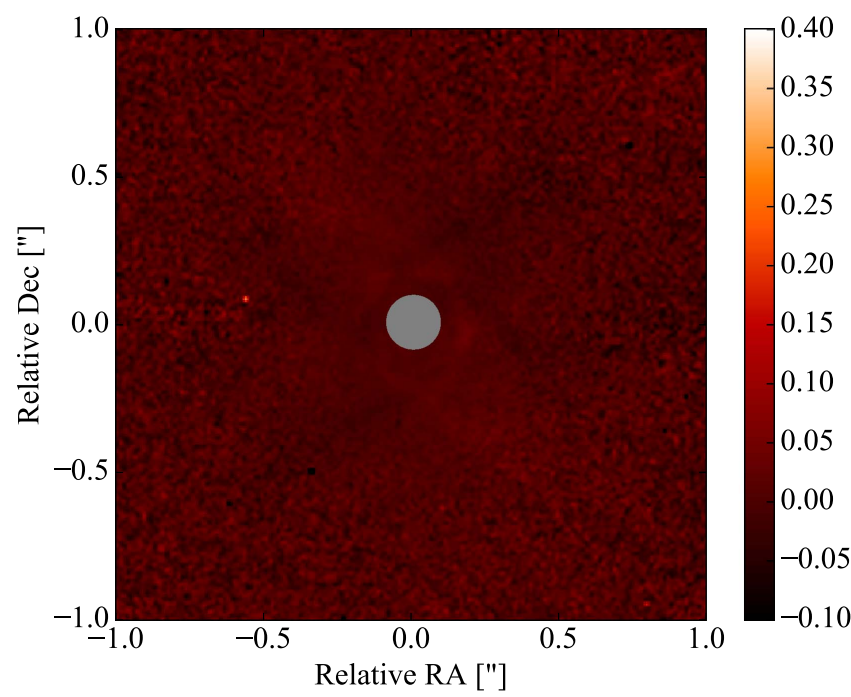

Figure 10. The $J$-band $U_{\phi} \times r^{2}$ image in linear scale. Each pixel is multiplied by the square root of its distance to the star, $r^{2}$, to compensate for the stellar illumination drop-off with radius. The normalization is similar to the $Q_{\phi}$ image, but the dynamical range of the color bar is adjusted. The region masked by the coronagraph is indicated by the gray circle. North is up, and east is toward the left.

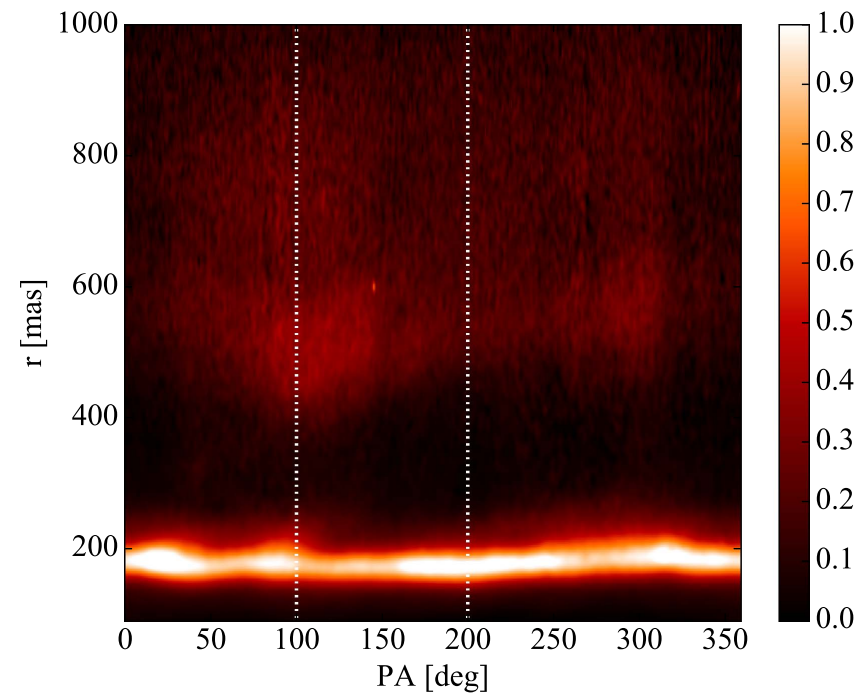

Figure 11. Polar map of the $Q_{\phi} \times r^{2}$ image in linear scale. The vertical dotted lines indicate $\mathrm{PA}=100^{\circ}$ and $200^{\circ}$. 
Appendix B

Ellipse Fitting

Table 1

Ellipse Parameters Fitted to the Two Rings and Gap \#2 in Our Scattered-light Images

\begin{tabular}{|c|c|c|c|}
\hline & Ring \#1 & Gap \#2 & Ring \#2 \\
\hline$\Delta$ R.A. (mas) & $28.4 \pm 5.5$ & $12.4 \pm 5.3$ & $4.4 \pm 2.9$ \\
\hline$\Delta$ Decl. (mas) & $18.9 \pm 5.7$ & $33.5 \pm 5.4$ & $5.3 \pm 2.8$ \\
\hline Semimajor axis (mas) & $536.4 \pm 18.2$ & $375.2 \pm 14.5$ & $173.8 \pm 9.1$ \\
\hline Semimajor axis (au) & $62.8 \pm 2.1$ & $43.9 \pm 1.7$ & $20.3 \pm 1.1$ \\
\hline Semiminor axis (mas) & $522.7 \pm 1.2$ & $365.6 \pm 1.5$ & $169.3 \pm 2.0$ \\
\hline Semiminor axis $(\mathrm{au})$ & $61.2 \pm 0.1$ & $42.8 \pm 0.2$ & $19.8 \pm 0.2$ \\
\hline
\end{tabular}

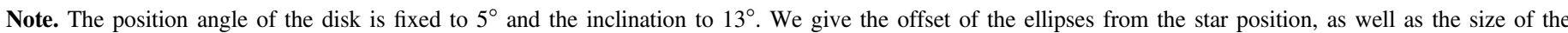
semimajor and semiminor axes for each fitted feature.

\section{Appendix C}

\section{Modeled 2D Disk Dust Density Distribution}

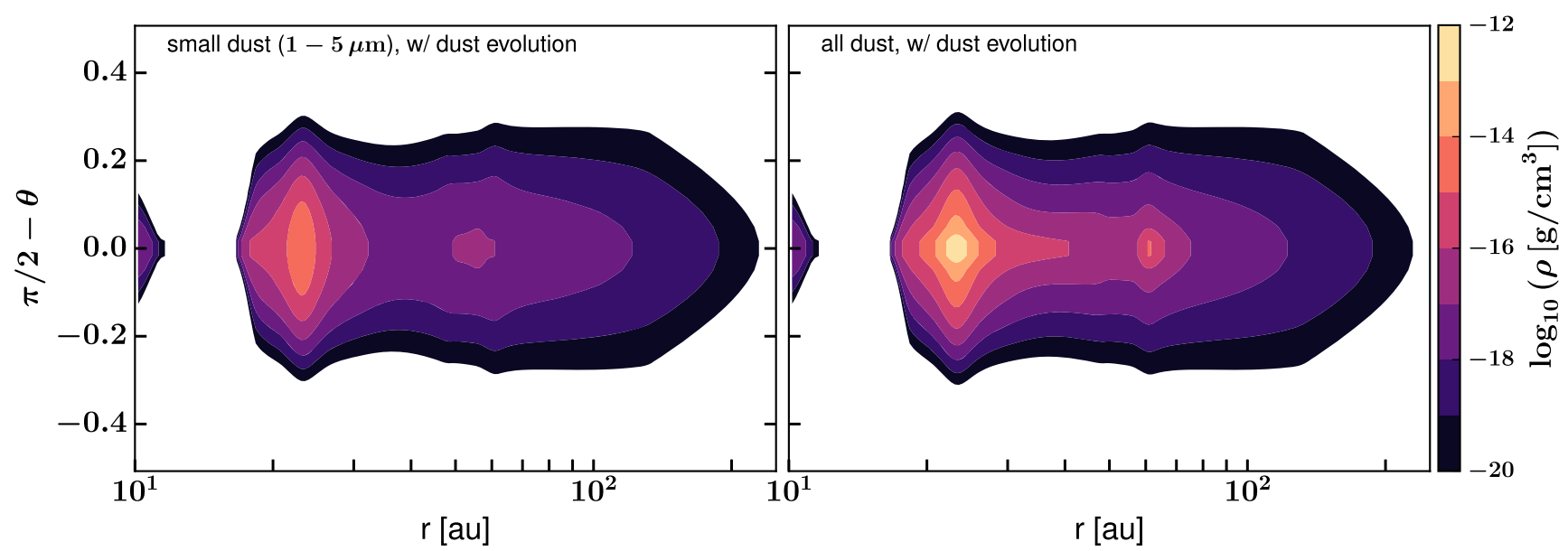

Figure 12. Vertical disk dust density structure assumed for the radiative transfer calculations following Equation (5) for an outer planet mass of $0.3 M_{\text {Jup. }}$ The cumulative density distributions for only small dust grains from 1 to $5 \mu \mathrm{m}$ (left) and for all dust grains (right) are shown. Note that the radial scale is logarithmic for a better visualization.

\section{Appendix D \\ Effect of Dust Evolution Timescale}
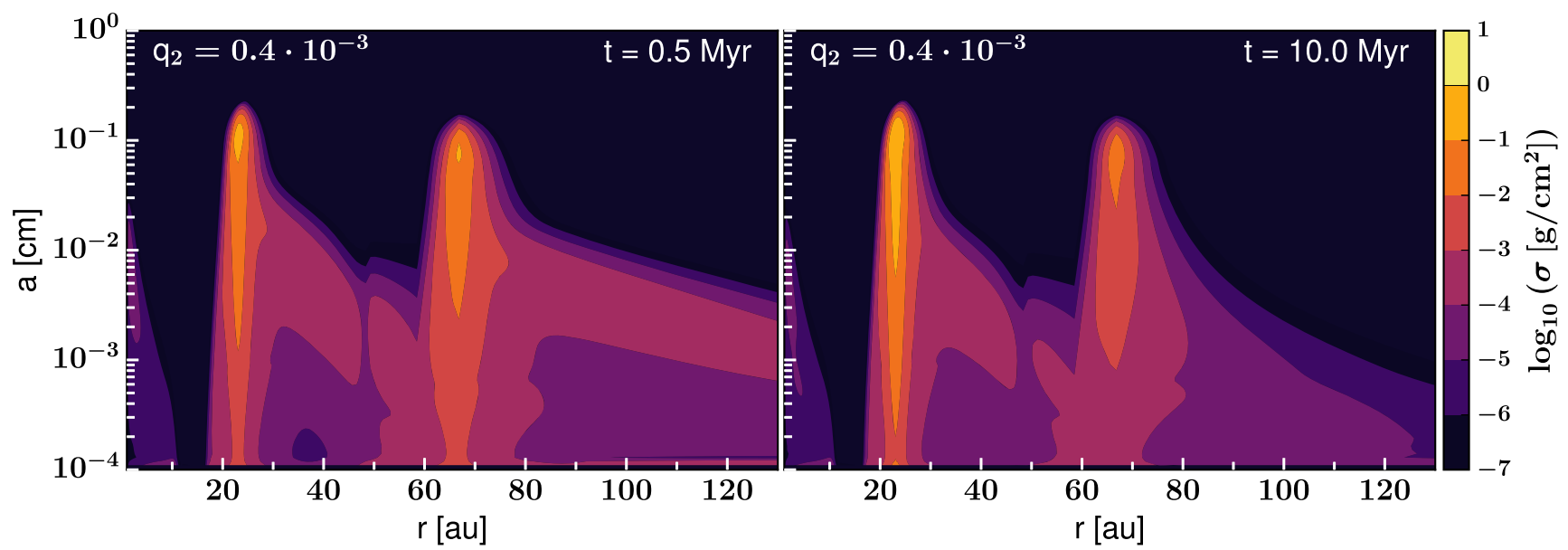

Figure 13. Vertically integrated dust density distribution after $0.5 \mathrm{Myr}$ (left) and $10 \mathrm{Myr}$ (right) of evolution, when two massive planets (3.5 and $0.3 M_{\mathrm{Jup}}$ ) are embedded in the disk at 14 and 53 au, respectively. 


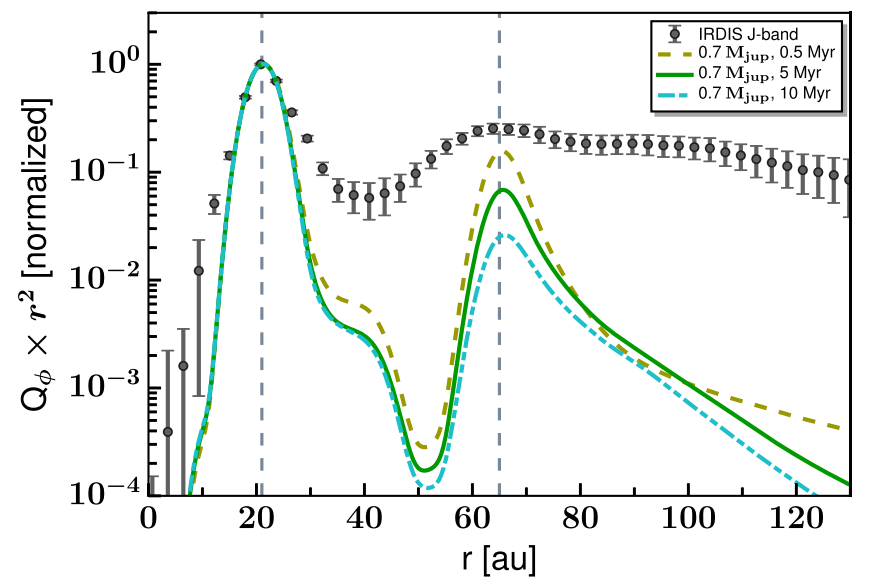

Figure 14. Comparison of surface brightness radial profiles of the $Q_{\phi} \times r^{2}$ model image at the $J$ band for different dust evolution timescales. The values are scaled by the square of the distance from the central star in order to compensate for the falloff of the stellar irradiation. The vertical dashed lines mark the positions of the brightness peaks.

\section{ORCID iDs}

M. Benisty (1) https://orcid.org/0000-0002-7695-7605 P. Pinilla (i) https://orcid.org/0000-0001-8764-1780 J. de Boer (1) https://orcid.org/0000-0002-1028-7522 A. Zurlo (i) https://orcid.org/0000-0002-5903-8316 T. Birnstiel (i) https://orcid.org/0000-0002-1899-8783 C. Dominik iㅣ https://orcid.org/0000-0002-3393-2459 S. Facchini (ib https://orcid.org/0000-0003-4689-2684 M. Janson (10 https://orcid.org/0000-0001-8345-593X A.-L. Maire (i) https://orcid.org/0000-0002-2591-4138 F. Ménard (i) https://orcid.org/0000-0002-1637-7393 M. Meyer (10 https://orcid.org/0000-0003-1227-3084 C. Pinte (i) https://orcid.org/0000-0001-5907-5179 S. P. Quanz (1) https://orcid.org/0000-0003-3829-7412 T. Stolker (ㄴ) https://orcid.org/0000-0002-5823-3072 G. van der Plas (1) https://orcid.org/0000-0001-5688-187X A. Baruffolo (1) https://orcid.org/0000-0002-1114-4355

\section{References}

ALMA Partnership, Brogan, C. L., Pérez, L. M., et al. 2015, ApJL, 808, L3 Andrews, S. M., Wilner, D. J., Zhu, Z., et al. 2016, ApJL, 820, L40 Apai, D., Pascucci, I., Brandner, W., et al. 2004, A\&A, 415, 671 Ataiee, S., Pinilla, P., Zsom, A., et al. 2013, A\&A, 553, L3 Avenhaus, H., Quanz, S. P., Schmid, H. M., et al. 2014, ApJ, 781, 87 Benisty, M., Juhasz, A., Boccaletti, A., et al. 2015, A\&A, 578, L6 Benisty, M., Stolker, T., Pohl, A., et al. 2017, A\&A, 597, A42 Béthune, W., Lesur, G., \& Ferreira, J. 2016, A\&A, 589, A87 Beuzit, J.-L., Feldt, M., Dohlen, K., et al. 2008, Proc. SPIE, 7014, 18 Biller, B. A., Males, J., Rodigas, T., et al. 2014, ApJL, 792, L22 Birnstiel, T., \& Andrews, S. M. 2014, ApJ, 780, 153

Birnstiel, T., Dullemond, C. P., \& Brauer, F. 2010, A\&A, 513, A79 Blondel, P. F. C., \& Djie, H. R. E. T. A. 2006, A\&A, 456, 1045

Boccaletti, A., Abe, L., Baudrand, J., et al. 2008, Proc. SPIE, 7015, 70151B

Brauer, F., Dullemond, C. P., \& Henning, T. 2008, A\&A, 480, 859

Canovas, H., Hardy, A., Zurlo, A., et al. 2017, A\&A, 598, A43

Canovas, H., Ménard, F., de Boer, J., et al. 2015, A\&A, 582, L7

Carrasco-González, C., Henning, T., Chandler, C. J., et al. 2016, ApJL, 821, L16

Christiaens, V., Casassus, S., Perez, S., van der Plas, G., \& Ménard, F. 2014, ApJL, 785, L12

Crida, A., Morbidelli, A., \& Masset, F. 2006, Icar, 181, 587

de Boer, J., Salter, G., Benisty, M., et al. 2016, A\&A, 595, A114 de Juan Ovelar, M., Pinilla, P., Min, M., Dominik, C., \& Birnstiel, T. 2016, MNRAS, 459, L85

Dipierro, G., \& Laibe, G. 2017, MNRAS, 469, 1932

Dipierro, G., Laibe, G., Price, D. J., \& Lodato, G. 2016, MNRAS, 459, L1

Dodson-Robinson, S. E., \& Salyk, C. 2011, ApJ, 738, 131

Dohlen, K., Langlois, M., Saisse, M., et al. 2008, Proc. SPIE, 7014, 70143

Dong, R., \& Fung, J. 2017, ApJ, 835, 146

Dong, R., Fung, J., \& Chiang, E. 2016, ApJ, 826, 75

Dong, R., Zhu, Z., \& Whitney, B. 2015, ApJ, 809, 93

Draine, B. T. 2003, ApJ, 598, 1026

Dullemond, C. P., Juhasz, A., Pohl, A., et al. 2012, RADMC-3D: A multipurpose radiative transfer tool, Astrophysics Source Code Library, ascl: 1202.015

Dunkin, S. K., Barlow, M. J., \& Ryan, S. G. 1997, MNRAS, 286, 604

Dzyurkevich, N., Turner, N. J., Henning, T., \& Kley, W. 2013, ApJ, 765, 114

Facchini, S., Birnstiel, T., Bruderer, S., \& van Dishoeck, E. F. 2017, A\&A, 605, A16

Fedele, D., Carney, M., Hogerheijde, M. R., et al. 2017, A\&A, 600, A72

Flock, M., Ruge, J. P., Dzyurkevich, N., et al. 2015, A\&A, 574, A68

Folsom, C. P., Bagnulo, S., Wade, G. A., et al. 2012, MNRAS, 422, 2072

Fung, J., Shi, J.-M., \& Chiang, E. 2014, ApJ, 782, 88

Fusco, T., Rousset, G., Sauvage, J.-F., et al. 2006, OExpr, 14, 7515

Fusco, T., Sauvage, J.-F., Petit, C., et al. 2014, Proc. SPIE, 9148, 1

Gaia Collaboration, Brown, A. G. A., Vallenari, A., et al. 2016, A\&A, 595, A2

Garufi, A., Meeus, G., Benisty, M., et al. 2017, A\&A, 603, A21

Garufi, A., Quanz, S. P., Avenhaus, H., et al. 2013, A\&A, 560, A105

Ginski, C., Stolker, T., Pinilla, P., et al. 2016, A\&A, 595, A112

Grady, C. A., Muto, T., Hashimoto, J., et al. 2013, ApJ, 762, 48

Grady, C. A., Schneider, G., Hamaguchi, K., et al. 2007, ApJ, 665, 1391

Kama, M., Bruderer, S., Carney, M., et al. 2016, A\&A, 588, A108

Kanagawa, K. D., Muto, T., Tanaka, H., et al. 2015, ApJL, 806, L15

Kataoka, A., Muto, T., Momose, M., et al. 2015, ApJ, 809, 78

Kataoka, A., Tsukagoshi, T., Momose, M., et al. 2016, ApJL, 831, L12

Kuhn, J. R., Potter, D., \& Parise, B. 2001, ApJL, 553, L189

Langlois, M., Dohlen, K., Vigan, A., et al. 2014, Proc. SPIE, 9147, 1

Lazareff, B., Berger, J.-P., Kluska, J., et al. 2017, A\&A, 599, A85

Ligi, R., Vigan, A., Gratton, R., et al. 2018, MNRAS, 473, 1774

Macías, E., Anglada, G., Osorio, M., et al. 2017, ApJ, 838, 97

Maire, A.-L., Langlois, M., Dohlen, K., et al. 2016, Proc. SPIE, 9908, 990834

Marino, S., Perez, S., \& Casassus, S. 2015, ApJL, 798, L44

Meeus, G., Pinte, C., Woitke, P., et al. 2010, A\&A, 518, L124

Menu, J., van Boekel, R., Henning, T., et al. 2015, A\&A, 581, A107

Momose, M., Morita, A., Fukagawa, M., et al. 2015, PASJ, 67, 83

Monnier, J. D., Harries, T. J., Aarnio, A., et al. 2017, ApJ, 838, 20

Mordasini, C., Alibert, Y., Klahr, H., \& Henning, T. 2012, A\&A, 547, A111

Mordasini, C., van Boekel, R., Mollière, P., Henning, T., \& Benneke, B. 2016, ApJ, 832, 41

Muto, T., Grady, C. A., Hashimoto, J., et al. 2012, ApJL, 748, L22

Okuzumi, S., Momose, M., Sirono, S.-i., Kobayashi, H., \& Tanaka, H. 2016, ApJ, 821, 82

Osorio, M., Anglada, G., Carrasco-González, C., et al. 2014, ApJL, 791, L36

Paardekooper, S.-J., \& Mellema, G. 2004, A\&A, 425, L9

Paardekooper, S.-J., \& Mellema, G. 2006, A\&A, 453, 1129

Panić, O., Hogerheijde, M. R., Wilner, D., \& Qi, C. 2008, A\&A, 491, 219

Pérez, L. M., Carpenter, J. M., Andrews, S. M., et al. 2016, Sci, 353, 1519

Petit, C., Sauvage, J.-F., Fusco, T., et al. 2014, Proc. SPIE, 9148, 0

Picogna, G., \& Kley, W. 2015, A\&A, 584, A110

Pinilla, P., Benisty, M., \& Birnstiel, T. 2012, A\&A, 545, A81

Pinilla, P., Birnstiel, T., \& Walsh, C. 2015a, A\&A, 580, A105

Pinilla, P., de Juan Ovelar, M., Ataiee, S., et al. 2015b, A\&A, 573, A9

Pinilla, P., Flock, M., Ovelar, M. d. J., \& Birnstiel, T. 2016, A\&A, 596, A81

Pinilla, P., Pohl, A., Stammler, S. M., \& Birnstiel, T. 2017, ApJ, 845, 68

Pohl, A., Kataoka, A., Pinilla, P., et al. 2016, A\&A, 593, A12

Pohl, A., Sissa, E., Langlois, M., et al. 2017, A\&A, 605, A34

Quanz, S. P., Avenhaus, H., Buenzli, E., et al. 2013, ApJL, 766, L2

Raman, A., Lisanti, M., Wilner, D. J., Qi, C., \& Hogerheijde, M. 2006, AJ, 131,2290

Rapson, V. A., Kastner, J. H., Millar-Blanchaer, M. A., \& Dong, R. 2015, ApJL, 815, L26

Reggiani, M., Quanz, S. P., Meyer, M. R., et al. 2014, ApJL, 792, L23

Ricci, L., Testi, L., Natta, A., et al. 2010, A\&A, 512, A15

Riviere-Marichalar, P., Merín, B., Kamp, I., Eiroa, C., \& Montesinos, B. 2016, A\&A, 594, A59

Rosotti, G. P., Juhasz, A., Booth, R. A., \& Clarke, C. J. 2016, MNRAS, 459, 2790 
Ruge, J. P., Flock, M., Wolf, S., et al. 2016, A\&A, 590, A17

Schmid, H.-M., Downing, M., Roelfsema, R., et al. 2012, Proc. SPIE, 8446, $84468 \mathrm{Y}$

Schmid, H. M., Joos, F., \& Tschan, D. 2006, A\&A, 452, 657

Seok, J. Y., \& Li, A. 2017, ApJ, 835, 291

Simon, J. B., \& Armitage, P. J. 2014, ApJ, 784, 15

Stammler, S. M., Birnstiel, T., Panić, O., Dullemond, C. P., \& Dominik, C. 2017, A\&A, 600, A140

Stolker, T., Dominik, C., Avenhaus, H., et al. 2016, A\&A, 595, A113

Strom, K. M., Strom, S. E., Edwards, S., Cabrit, S., \& Skrutskie, M. F. 1989, AJ, 97, 1451

Takahashi, S. Z., \& Inutsuka, S.-i. 2014, ApJ, 794, 55

Tang, Y.-W., Guilloteau, S., Dutrey, A., et al. 2017, ApJ, 840, 32

Thalmann, C., Schmid, H. M., Boccaletti, A., et al. 2008, Proc. SPIE, 7014, $70143 \mathrm{~F}$
Tsukagoshi, T., Nomura, H., Muto, T., et al. 2016, ApJL, 829, L35

van Boekel, R., Henning, T., Menu, J., et al. 2017, ApJ, 837, 132

van der Plas, G., Wright, C. M., Ménard, F., et al. 2017, A\&A, 597, A32

Wagner, K. R., Sitko, M. L., Grady, C. A., et al. 2015, ApJ, 798, 94

Warren, S. G., \& Brandt, R. E. 2008, JGRD, 113, D14220

Williams, J. P., \& Cieza, L. A. 2011, ARA\&A, 49, 67

Yang, H., Li, Z.-Y., Looney, L., \& Stephens, I. 2016, MNRAS, 456, 2794

Youdin, A. N. 2011, ApJ, 731, 99

Zhang, K., Blake, G. A., \& Bergin, E. A. 2015, ApJL, 806, L7

Zhu, Z., Nelson, R. P., Dong, R., Espaillat, C., \& Hartmann, L. 2012, ApJ, 755,6

Zhu, Z., Nelson, R. P., Hartmann, L., Espaillat, C., \& Calvet, N. 2011, ApJ, 729,47

Zubko, V. G., Mennella, V., Colangeli, L., \& Bussoletti, E. 1996, MNRAS, 282, 1321 